\title{
La navigation en Loire au XVIIIe siècle vue à travers les procès-verbaux d'avaries
}

\section{Emmanuel Brouard}

\section{OpenEdition}

Journals

Édition électronique

URL : http://journals.openedition.org/abpo/1116

DOI : $10.4000 / a b p o .1116$

ISBN : 978-2-7535-1499-7

ISSN : 2108-6443

Éditeur

Presses universitaires de Rennes

\section{Édition imprimée}

Date de publication : 20 septembre 2005

Pagination : 37-69

ISBN : 978-2-7535-0201-7

ISSN : 0399-0826

Référence électronique

Emmanuel Brouard, «La navigation en Loire au XVIIII siècle vue à travers les procès-verbaux d'avaries », Annales de Bretagne et des Pays de l'Ouest [En ligne], 112-3 | 2005, mis en ligne le 20 septembre 2007, consulté le 19 avril 2019. URL : http://journals.openedition.org/abpo/1116; DOI : $10.4000 / a b p o .1116$ 


\title{
La navigation en Loire au XVIII ${ }^{\mathrm{e}}$ siècle vue à travers les procès-verbaux d'avaries
}

\author{
Emmanuel BROUARD \\ étudiant, université de Poitiers
}

$\mathrm{Au} \mathrm{XVIII}{ }^{\mathrm{e}}$ siècle la Loire était un axe commercial très important emprunté par des milliers de bateaux entre Nantes et la haute Loire. Le sucre d'Amérique et la morue séchée, embarqués à Nantes, croisaient l'ardoise et le blé d'Anjou, le charbon et le bois du Massif central. Mais les tempêtes, les débris encombrant le fleuve, les abords dangereux des ponts, tous ces éléments provoquaient de nombreux accidents. Comme les propriétaires des marchandises embarquées pouvaient être tentés de demander le remboursement des marchandises endommagées, les "voituriers par eaux ", propriétaires des bateaux, faisaient dresser des procès-verbaux par des notaires des environs pour se justifier. Ces procèsverbaux sont une précieuse source d'informations sur la navigation en Loire, décrite par ceux qui la pratiquaient. Nous en avons étudié 231, rédigés de 1689 à 1791, entre Ingrandes et Montsoreau dans l'actuel département du Maine-et-Loire ${ }^{1}$. Nous verrons d'abord ce que ces documents nous apprennent sur les conditions de navigation : les bateaux utilisés, les méthodes de navigation vers l'aval et vers l'amont, les équipages et l'irrégularité des temps de trajet. Ensuite nous aborderons les accidents à l'origine des procès-verbaux, ainsi que les conditions de sauvetage des bateaux et des marchandises ${ }^{2}$.

1. La majorité de ces procès-verbaux ont été rédigés entre Angers et Saumur après 1750. 200 sont liés à des avaries, 27 à des retards de livraison et quatre actes concernent des avaries survenues aux seules marchandises. L'étude de ces sources s'est faite par sondages dans le fond notarial des Archives départementales de Maine-et-Loire, en soussérie $5 \mathrm{E}$. Nous avons aussi utilisé des contrats de vente et de location de bateaux ainsi que des inventaires après décès de mariniers.

2. Ces documents sont aussi intéressants pour l'étude du commerce fluvial. Ils nous renseignent sur les noms et résidences des voituriers et des marchands ainsi que sur la nature, la provenance et la destination des marchandises. Mais nous n'aborderons pas ici le sujet des échanges commerciaux, nous cantonnant aux conditions de navigation. 


\section{La navigation en Loire}

\section{Les bateaux, l'adaptation au débit du fleuve et la protection des marchandises}

Les bateaux de Loire étaient effilés et légers pour remonter le courant, sans quille et à faible tirant d'eau pour passer sur les bancs de sable ${ }^{3}$. Les trois principaux types de bateaux rencontrés sur la Loire angevine étaient le chaland, la toue et la sapine.

Le chaland était un grand bateau de Loire, le plus utilisé sur cette partie du fleuve ${ }^{4}$. Construit en chêne, le chaland de Loire mesurait de 24 à 30 mètres de longueur (12 à 15 toises), généralement autour de 28 mètres, pour 3,20 mètres à 3,90 mètres de large (10 à 12 pieds) ${ }^{5}$. Il était souvent accompagné d'un bateau de petite taille appelé " toue de bord ". Facilement manœuvrable, elle servait à accéder au chaland et à reconnaître le chenal. Des toues plus grandes existaient aussi mais elles restaient généralement d'une taille inférieure aux chalands mêmes si certaines atteignaient une longueur comparable ${ }^{6}$. Elles s'en distinguaient par une plus grande largeur $^{7}$. Des bateaux de petite taille appelés pillards étaient parfois assimilés aux toues, mais ils devaient s'en distinguer par des spécificités que je n'ai pu déterminer ${ }^{8}$.

La sapine était, après le chaland, l'autre grand bateau de Loire. Sur les 231 procès-verbaux étudiés, 25 concernent explicitement des sapines. De taille très variable, mais souvent plus petits que les chalands, ces bateaux étaient fabriqués sur la haute Loire et l'Allier. On les appelait aussi saintrambertes, salembardes (du nom de la ville de Saint-Rambert près de SaintÉtienne) ou auvergnates. Les sapines étaient fabriquées en sapin et coûtaient bien moins cher que les chalands, mais leur espérance de vie était

3. Voir les travaux de François BEAUDOUIN sur les bateaux de Loire, notamment " La marine de Loire et son chaland ", Bulletin de l'Association des Amis du Musée de la Batellerie, Conflans-Sainte-Honorine, $\mathrm{n}^{\circ} 12$, juin 1984.

4. Dans la majorité des procès-verbaux, le mot " bateau " domine, mais le mot " chaland " est généralement employé dans les actes de vente et dans les contrats de location de bateaux, souvent plus détaillés.

5. Nous avons observé les mêmes dimensions dans des contrats de vente de bateaux en Anjou, et Paul Chaussard donne des dimensions comparables pour les grands bateaux ou chalands fabriqués près de Digoin dans la seconde moitié du XVII ${ }^{\mathrm{e}}$ siècle : entre 12 et 16 toises (23 à 31 mètres), souvent 13 ou 14. ChausSARd, Paul, La Marine de Loire, Éd. Charroux, Cahiers du Bourbonnais, 1998, p. 37.

6. Arch. dép. de Maine-et-Loire, 5E 90/605, notaire Fruslon des Ponts-de-Cé, procèsverbal du 18 mai 1765 : naufragés sauvés par d'autres mariniers grâce à un " petit bateau vulgairement appellé toue ". 5E 90/610, notaire Fruslon des Ponts-de-Cé, procès-verbal du 23 octobre 1772 : "trois petits batteaux nommés grande toues ".

7. Exemple : «Une grande toue ou bateau de chesne d'environ dix pieds deux pouces de largeur dans le milieu sur environ quarante pieds de longueur " : 13 mètres sur 3,25 mètres, soit la largeur d'un chaland de 24 mètres (Arch. dép. de Maine-et-Loire, 5E 3/86, notaire Davy de Chalonnes, procès-verbal du 12 juillet 1777).

8. Arch. dép. de Maine-et-Loire, 5E 13/446, notaire Guillemet de Saint-Martin-de-laPlace, procès-verbal du 5 avril 1767 : une toue " appellée pillart ". 
plus limitée. Elles servaient surtout à transporter vers Orléans et vers Paris (par le canal de Briare) du charbon, du bois et du vin en grandes quantités. Une minorité allait jusqu'à Nantes, Angers ou Tours. Comme la navigation vers la haute Loire était difficile et coûteuse, et le fret limité, les sapines n'étaient pas destinées à remonter le fleuve jusqu'à leur lieu de fabrication. Les voituriers de la haute Loire revendaient leurs sapines en aval et revenaient chez eux à pied. Certaines sapines étaient alors déchirés (démantelés), en particulier quand leur voyage les avait conduit à Paris, mais les autres entraient dans le marché de l'occasion ${ }^{9}$. L'étude des procès-verbaux montre que les sapines connaissaient alors une seconde vie en aval d'Orléans, entre les mains de nouveaux propriétaires, et qu'elles servaient à de multiples trajets tant vers l'aval que vers l'amont ${ }^{10}$. En effet, sur vingt-quatre procès-verbaux, seize concernent des sapines descendant la Loire, et huit des sapines la remontant ${ }^{11}$. L'une d'elles, chargée d'ardoises aux Pont-de-Cé, est même destinée à Paris ${ }^{12}$. Seuls trois propriétaires de sapines sur vingt-cinq viennent de la haute Loire, les autres sont des Orléanais, Angevins, Tourangeaux et Poitevins qui ont dû acheter ces bateaux au terme de leurs premiers voyages ${ }^{13}$.

Selon François Billacois, le déséquilibre du trafic entre la haute Loire et la Loire moyenne entraîna une spécialisation de la haute Loire dans la fabrication des bateaux entre le milieu du XVII ${ }^{\mathrm{e}}$ siècle et le début du XVIII ${ }^{\mathrm{e}}$ siècle, cette situation se maintenant jusqu'au déclin de la navigation en Loire au $\mathrm{XIX}^{\mathrm{e}}$ siècle. Les voituriers de l'amont revendaient leurs sapines à des prix que ne pouvaient concurrencer les charpentiers en bateaux de l'aval ${ }^{14}$. L'habitude fut prise d'acheter les bateaux de l'amont, y compris les bateaux en chêne. Ainsi, en 1733, la municipalité d'Orléans et les négociants de la ville remarquent dans un mémoire qu'il " ne se construit point de bateaux à Orléans : les mariniers achètent ceux de Roanne dont ils se servent pour

9. BILLACOIS, François "La batellerie de la Loire au XVII ${ }^{\mathrm{e}}$ siècle ", Revue d'Histoire Moderne et Contemporaine, 1964, p. 172.

10. Un voiturier de Rochefort affirmait d'ailleurs que sa sapine victime d'un accident était " toute neuve " car elle "n'avait point servie qu'à venir de l'endroit ou elle avoit été construite " (Arch. dép. de Maine-et-Loire, 5E 52/79, notaire Moron de Rochefort-sur-Loire, procès-verbal du 9 mai 1775).

11. Nous connaissons le sens du déplacement de vingt-quatre sapines sur vingt-cinq. Treize sur vingt vont à Nantes, trois à Orléans, une à Paris, les autres à Angers, Saumur et Tours. Cinq destinations sont inconnues. Les sapines viennent du Saumurois (5), d'Angers ou de ses environs (5), de Châtellerault (2), de la haute Loire (3), de Tours (1), et de Nantes (4). Pour six procès-verbaux nous ignorons le lieu de départ, mais d'après certains éléments ils semblent venir de la Loire Moyenne ou de la basse Loire. Nous disposons aussi de deux contrats de location de sapines, tous deux pour remonter la Loire.

12. Arch. dép. de Maine-et-Loire, 5E 37/213, notaire Gaillard de Montsoreau, procès-verbal du 23 septembre 1762 .

13. Cinq voituriers résidaient entre Angers et Ingrandes, six en Saumurois, six entre Blois et Chinon, deux à Châtellerault. Soit seize à Blois ou en aval. Dans ces actes, seuls trois voituriers sur vingt-cinq habitaient en haute Loire (Nevers, Roanne, Saint-Étienne) et trois à Orléans.

14. BiLlaCoIs, François, op. cit., p. 171. 
descendre aux pays bas ${ }^{15}$ " et Paul Chaussard signale que dans la seconde moitié du XVII ${ }^{\mathrm{e}}$ siècle des charpentiers en bateaux de la région de Digoin travaillaient souvent pour des voituriers d'Orléans et de Touraine ${ }^{16}$. Enfin Henri Chevaleau, ancien marinier et garde-port né en 1791, cite dans la première moitié du XIX ${ }^{\mathrm{e}}$ siècle vingt-sept lieux de construction de bateaux, dont vingt en amont de Nevers, sur la Loire et l'Allier, contre un seul en aval de Tours, près d'Ingrandes ${ }^{17}$.

Effectivement, soit les charpentiers de la Loire angevine travaillaient surtout à la réparation des bateaux, soit leur activité de fabrication est trop marginale pour apparaître dans les documents. Nous disposons pour le $\mathrm{XVIII}^{\mathrm{e}}$ siècle de trente-quatre contrats de vente de bateaux et aucun charpentier n'y figure. Ces contrats concernent tous des reventes d'occasion entre voituriers, angevins pour la plupart. Des chantiers actifs existaient par contre sur la Mayenne à la veille de la Révolution, autour de GrezNeuville et du Lyon d'Angers, mais on y construisait surtout des bateaux particuliers à la Mayenne, appelés hannequins. Il est vrai que des gabarres apparemment identiques aux chalands sortaient aussi de ces chantiers. Un document de 1774 signale d'ailleurs la vente d'un chaland par un charpentier en bateaux de Grez-Neuville à un voiturier de Chalonnes-sur-Loire ${ }^{18}$. Mais, dans l'ensemble, les charpentiers de la Mayenne, semblaient surtout fournir les voituriers locaux, et majoritairement en hannequins ${ }^{19}$. Ils ne constituaient qu'une source d'approvisionnement occasionnelle pour les voituriers de la Loire.

Les grands bateaux, chalands ou sapines, avaient une hauteur de bord d'un mètre trente (quatre pieds) et un tirant d'eau de soixante-cinq centimètres (deux pieds) ${ }^{20}$. Ce faible tirant d'eau était nécessaire pour naviguer

15. Mémoire disparu, anciennement conservé aux Archives départementales du Loiret (C331), et heureusement cité par Roger Dion dans « Orléans et l'ancienne navigation de la Loire ", Annales de géographie, 1938.

16. Chaussard, Paul, op. cit., p. 47. Dans les exemples donnés, sur treize acheteurs de bateaux, quatre sont d'Orléans et trois de Touraine.

17. "Le manuscrit Chevaleau. 1839-1860. $1^{\text {re }}$ partie : l'homme, la rivière et les bateaux ". Bulletin de l'Association des Amis du Musée de la Marine de Loire (Châteauneuf-sur-Loire), $\mathrm{n}^{\circ} 74$, avril 1995 , carte p. 65 bis.

18. Arch. dép. de Maine-et-Loire, 5E 3/48, notaire Burgevin de Chalonnes, inventaire après décès des biens de Pierre Robin voiturier par eau de Chalonnes, 2 et 3 novembre 1774 .

19. Sur douze bateaux construits entre 1778 et 1787 par les charpentiers Louis et Jean Gautier du Lion-d'angers, neuf sont des hannequins et trois des gabarres, destinés à des voituriers de la Mayenne et d'Angers (Arch. dép. de Maine-et-Loire, 5E 12/23 à 5E 12/32, notaire Gilbert de Pontchâteau du Lion-d'Angers). Ces deux types de bateaux se retrouvent aussi dans les productions des autres charpentiers.

20. Arch. dép. de Maine-et-Loire, 5E 19/52, notaire Hullin de Savennières, procès-verbal du 17 mai 1719, « ledit bateau etant chargé de lad. marchandise pouvoit tenir deux pied de font dans l'eau ». 5E 19/10, notaire Dertron de Savennières, procès-verbal du 26 septembre 1773, " un de ses bateaux qui tenait vingt trois pouces d'au passant par le milieu du ruau [...] avoit rencontré un arbre de la grosseur d'un quart de pipe qui netoit couvert que de dix huit pouces deau ". Dans les procès-verbaux, les obstacles immergés à l'origine des naufrages se trouvent en général à un pied et demi de fond. 
en Loire à cause de la faible profondeur du chenal pendant une grande partie de l'année. Malgré cela des voituriers restaient souvent bloqués par les basses eaux pendant des semaines ${ }^{21}$. Pour naviguer plus longtemps, ils limitaient l'été la charge de leurs bateaux, remontant ainsi la ligne de flottaison ${ }^{22}$. En 1802, le préfet de Maine-et-Loire signale que

" dans les quatre mois les plus propres à la navigation, c'est-à-dire depuis brumaire jusqu'à germinal [ 22 octobre-19 avril], les plus forts bateaux qui partent de Nantes pour Saumur, Tours et Orléans, ne tirent pas plus de 73 centimètres, autrement ils courraient le risque de s'attérer dans le trajet. Il y a trois autres mois où l'on navigue depuis 65 centimètres jusqu'à 487 millimètres. Tout le reste de l'année, la navigation n'est que précaire, et se fait par des bateaux légers appelés sapines, ou autres batteaux n'ayant que le tiers ou moitié de la charge ordinaire ${ }^{23}$ ".

Les voituriers étaient donc pris entre la nécessité de charger autant que possible leurs bateaux, et celle de maintenir un tirant d'eau réduit pour éviter de s'engraver sur un banc de sable. Quand le chenal se rétrécissait les voituriers faisaient couramment précéder leurs bateaux d'une toue chargée de reconnaître le chemin, d'autant que le balisage était souvent mal fait. On parlait alors de " faire la toue ${ }^{24}$ ". Dans les procès-verbaux la charge portée par les bateaux est généralement comprise entre dix et trente tonnes, le maximum relevé étant de quarante-cinq tonnes. Les valeurs relevées ne correspondent pas à la capacité maximale des bateaux, car le volume et la masse de la cargaison dépendaient du contrat passé par le voiturier, du type de marchandises et de la limite imposée au tirant d'eau par le débit du fleuve.

Les marchandises étaient habituellement soutrées et cabanées. Le soutrage ou soutrait était une couche de paille, de fagots ou de foin d'une trentaine de centimètres, parfois recouverte de nattes, qui isolait la marchandise du fond du bateau ${ }^{25}$. En effet, ce fond était rarement sec, soit à cause

21. En 1762 six bateaux chargés de 293 milliers d'ardoises doivent attendre plus de trois semaines devant l'île de Montsoreau " n'ayant pu trouver l'eau assez profonde pour rendre leurs batteaux a leur destination " (Arch. dép. de Maine-et-Loire, 5E 37/213, notaire Gaillard de Montsoreau, procès-verbal du 23 septembre 1762).

22. Dans un procès-verbal un " challan neuf " porte 2100 boisseaux de charbon de terre mais " si l'eau croissait ils chargeraient davantage leurs bateaux netant chargés qu'à moitié raport aux basses eaux " (Arch. dép. de Maine-et-Loire, 5E 3/89, notaire Davy de Chalonnes, procès-verbal du 27 juin 1786).

23. Arch. dép. de Maine-et-Loire, 6M (ancien 54M1, reclassé). Essais de statistique du département, présenté au ministre de l'Intérieur le 30 fructidor an 10 (1802).

24. Un bateau heurte un arbre en suivant « les balises que sa toux qui vat devant avoit marqué et par le fil de l'eau ou les bateaux ont coutume de passer et ou plusieurs venoient de passer " (Arch. dép. de Maine-et-Loire, 5E 90/658, notaire Foucher des Ponts-de-Cé, procès-verbal du 26 juin 1782). Cette pratique apparaît dans cinq procès-verbaux : une fois en mars, deux fois en juin et deux fois en août, donc surtout en période de basses eaux.

25. "La marchandise etait posée dans le challand sur un soutrait de dix à onze pouces de hauteur recouvert de dix nattes sur lesquels la marchandise paraît avoir eté chargée avec precaution en cinq mottes et en grenis et qu'elle est couverte soigneusement de trois à quatre nattes " (Arch. dép. de Maine-et-Loire, 5E 8/65, notaire Brevet d'Angers, procès-verbal du 26 janvier 1789). 
Figure 1 - Vue de Blois en 1784 par Gauché d'après C. F. Mandard

(Source : Musée des Beaux-arts de Blois, Tiré de Bateliers sur la Loire, par Françoise de Person, p. 136-137)

Au premier plan on voit un chaland de Loire à quai. À l'avant sont posés les bâtons de quartier qui servent à diriger le bateau en s'appuyant sur le fond du fleuve. À l'arrière-plan deux trains de bateaux franchissent le pont.

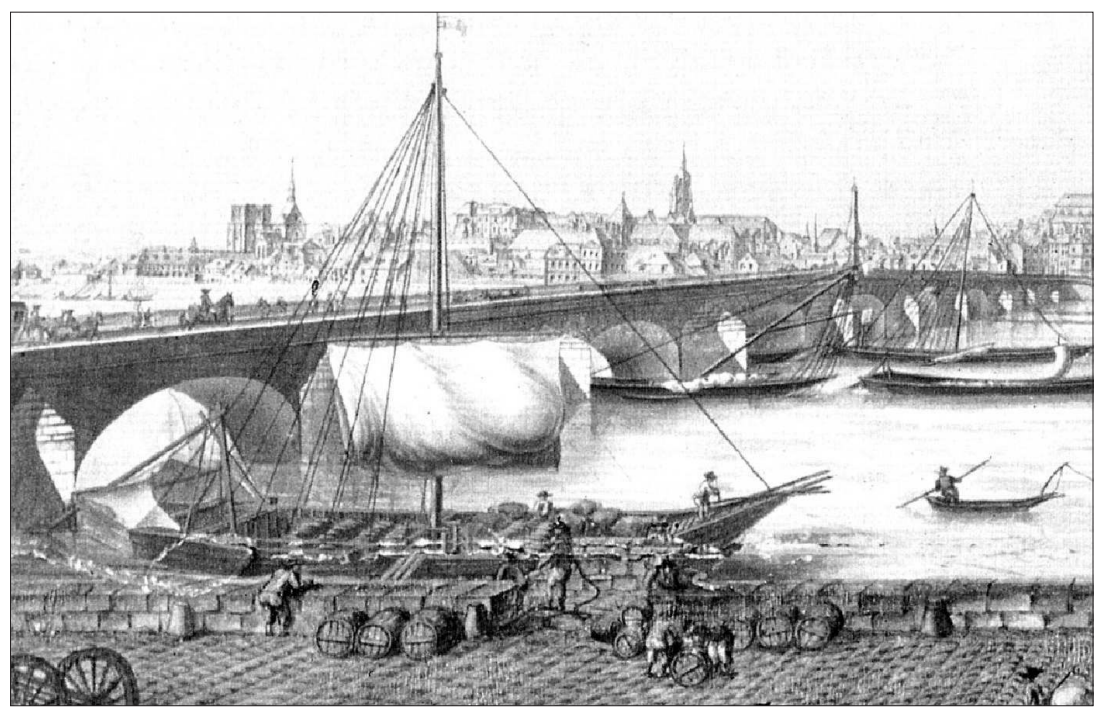

des pluies, soit à cause de l'eau qui s'infiltrait entre les planches des bordages. Les bateaux devaient d'ailleurs être vidés régulièrement ${ }^{26}$. Le cabanage était constitué de planches dont on recouvrait la marchandise pour la protéger de la pluie. Cela explique la présence de grandes quantités de planches de peuplier et de saule dans les inventaires après décès des voituriers ${ }^{27}$, alors que ces planches ne pouvaient pas servir à la construction des bateaux. Deux procès-verbaux signalent aussi l'utilisation de trois à cinq couches de nattes sur les marchandises. Quand le voyage s'éternisait et que le temps restait pluvieux, les marchandises pouvaient, malgré ces précautions, prendre l'humidité. Il arrivait que la pluie, la grêle ou la tempête soulèvent le cabanage ${ }^{28}$. Le froid était aussi susceptible de causer des

26. Suite à une avarie, un voiturier de Chalonnes affirme que son bateau " etait un des meilleurs de Chalonnes si vray qu'on auroit pu estre deux a trois jours sans jette leau quoy que chargé " (Arch. dép. de Maine-et-Loire, 5E 3/89, notaire Davy de Chalonnes, procès-verbal du 27 juin 1786).

27. Inventaires des biens meubles ayant appartenus à un individu ou à une communauté utilisés pour les partages entre les héritiers. Les inventaires concernant des familles de voituriers par eau nous fournissent des descriptions détaillées de bateaux, et du matériel les qui accompagnait : cordages, outils, voiles, etc.

28. "Les pluies gresle qui tomboient en abondance ont fait enlever les planches qui cabanoient les dittes farines et bled " (Arch. dép. de Maine-et-Loire, 5E 90/658, notaire Foucher des Ponts-de-Cé, procès-verbal du 27 mars 1783). 
dégâts aux marchandises, et les voituriers étaient mal armés pour le combattre. Les tonneaux de vin étaient particulièrement sensibles aux grands froids. De la glace se formait entre les douves des tonneaux et les écartait, le vin sortait alors par les fentes, gelait et les écartait davantage. Les mariniers étaient obligés de couper à la hache la glace qui se formait sur les barriques et de mettre en dessous des récipients pour récupérer le vin.

\section{Les techniques utilisées : \\ trains de bateaux vers l'amont et couplages vers l'aval}

Les vents d'ouest dominants permettaient de remonter la Loire à la voile jusqu'à Orléans. Les voituriers attachaient leurs bateaux à la file, formant un train de bateaux ou équipe. Le premier bateau du train était appelé chaland maté, le second tirot, et le troisième soubre ou second tirot. Ensuite venaient de petits bateaux non mâtés appelés allèges. Il semble que l'utilisation d'un train de plusieurs bateaux permettait d'économiser du personnel. Le tirot pouvait être un chaland classique, une sapine, une toue ou un pillard. Le mot tirot était aussi utilisé dans le sens de bateaux à la suite des autres, à l'arrière, à tirot, même s'il était à la quatrième ou à la cinquième place ${ }^{29}$.

Les chalands mâtés, qui portaient le plus grand mât ainsi qu'un énorme gouvernail appelé pautre, devaient être à la fois grands et résistants ${ }^{30}$. Les voituriers qui possédaient plusieurs bateaux aux caractéristiques différentes fixaient la place de chacun dans leurs trains en fonction de leur poids, de leur taille ou de leur solidité. C'est pourquoi les chalands, tirots et soubres étaient en général clairement différenciés dans les inventaires après décès.

On pourrait s'attendre à ce que, dans un train, le chaland porte la charge la plus lourde, étant le plus grand bateau. Mais deux procès-verbaux de 1790 et 1791 concernant des trains de bateaux chargés de sel nous donnent la répartition de la charge, et l'on découvre grâce à ces documents que les bateaux de tête portaient une charge relativement réduite. Cette répartition devait aussi permettre aux premiers bateaux, plus exposés au courant, de maintenir une distance suffisante par rapport aux suivants, et de rendre le train plus manœuvrable ${ }^{31}$.

29. « Le dernier desdits quatre bateaux etant le dernier a tirot ", « le dernier bateau etant a tirot des trois autres " Arch. dép. de Maine-et-Loire, 5E 13/58, notaire Legeay des Rosiers, procès-verbal du 23 décembre 1741.

30. «Le bateau de tête, ou de mât est une construction beaucoup plus forte parce qu'il porte le premier mât et ouvre le chemin pour le passage de l'équipe. " Extrait d'un " mémoire concernant la navigation des rivières sur lesquelles les entrepreneurs de la voiture du sel font le fournissement des greniers de grande gabelle " de 1785 (Archives Nationales, G1/97) publié sous le titre : "La navigation sur la Loire et ses affluents vers 1785 ", dans Annales de Bretagne, 36, 1, 1924-1925, p. 76-95. Aussi publié dans l'Anjou historique de janvier 1927.

31. Arch. dép. de Maine-et-Loire, 5E3/93, notaire Davy de Chalonnes, procès-verbal du 22 mars 1791. 5E 108/8, notaire Gazeau de Saint-Florent-le-Vieil, procès-verbal du 11 mars 1790. 
Procès-verbal de 1790 :

$1^{\mathrm{er}}$ bateau

8 muids

$2^{\mathrm{e}}$ et $3^{\mathrm{e}}$

$4^{\mathrm{e}}$

18 muids moins 24 quarteaux (total des deux)

10 muids 24 quarteaux

Procès-verbal de 1791 :

$1^{\text {er }}$ bateau

7 muids $1 / 2$ et 33 quarts

$2^{\mathrm{e}}$ (tirot)

8 muids $1 / 2$

$3^{\mathrm{e}}$

10 muids 18 quarts

$4^{\mathrm{e}}$

11 muids $1 / 2$

$5^{\mathrm{e}}$

12 muids $1 / 2$

$6^{\mathrm{e}}$

vide

Figure 2 - Détail d'un plat de Nevers, musée de Châteauneuf-sur-Loire (Source : VILIERS, Patrick, SENOTIER, Annick, Une histoire de la marine de Loire, p. 31) Sur ce dessin seul le chaland mâté, à la tête du train est équipé d'une " pautre " ou gouvernail. Sur ce même bateau deux voituriers manient des bâtons de quartier.

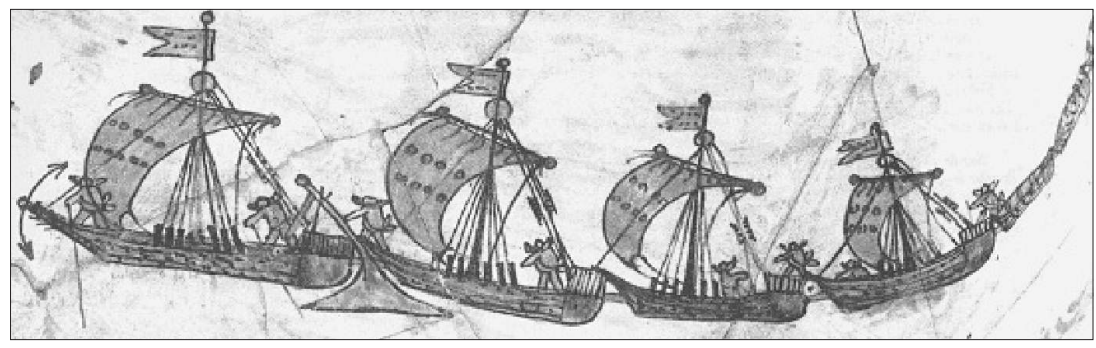

Dans un grand train, tous les bateaux n'étaient pas systématiquement dotés d'un mât. La multiplication des éléments " moteurs " du train devait rendre la manœuvre plus délicate et demander plus de personnel. Dans un train de deux bateaux, chaland et tirot étaient habituellement matés, sauf si le tirot était une sapine. Mais à partir de trois bateaux, l'un d'eux pouvait ne pas l'être, et sur quatre bateaux, généralement deux ou trois étaient matés. Dans un inventaire de biens après décès daté de 1758, sur un train de sept bateaux, seuls les quatre premiers l'étaient ${ }^{32}$. Les sapines étaient rarement matées même quand elles faisaient partie d'un train remontant

Le muid utilisé est peut-être celui de Paris, de 24,97 hectolitres, utilisé pour la gabelle (Françoise de Person : Bateliers et contrebandiers du sel. XVII -XVIII siècle, 1999). Un troisième procès-verbal donne des informations partielles pour un train constitué de 6 bateaux chargés de 340 milliers d'ardoises, le premier bateau étant chargé de 31 milliers soit $9 \%$ de la charge totale, alors qu'il en aurait porté $17 \%$ si la charge avait été également répartie. (Arch. dép. de Maine-et-Loire, 5E 17/205, notaire Jeffray de la Daguenière, procès-verbal du 30 juillet 1787). Dans d'autres actes, le chaland porte la plus grande charge, mais la grande différence de taille avec les autres bateaux ou leur absence de mât l'explique facilement.

32. Arch. dép. de Maine-et-Loire, 5E 90/601, notaire Fruslon des Ponts-de-Cé, inventaire Germain Moreau du 18 août 1758. 
le fleuve. D'une part, la pose d'un mât n'était pas prévue lors de leur fabrication, et d'autre part elles étaient plus fragiles que les bateaux en chêne ${ }^{33}$.

Le mât du chaland était amovible pour permettre le passage des ponts. On le baissait vers l'avant grâce à un gros treuil appelé guindas situé à l'arrière du bateau. Dans la plupart des procès-verbaux et des inventaires après décès, la taille des voiles est indiquée en nombre de toiles (voiles de 8 toiles, de 13 toiles, etc.) et quelquefois en aunes, le mot toile désignant la largeur d'un coupon de toile, soit une aune (1,19 m). Certains inventaires indiquent à la fois la superficie des voiles en aunes au carré, et le nombre de toiles, ce qui permet de connaître leurs proportions ${ }^{34}$. Ces voiles étaient rectangulaires, plus hautes que larges dans un rapport d'environ deux pour trois. Un chaland de 24 à 30 mètres (12 à 15 toises) portait une voile de 8 à 13 toiles de large, souvent 10 toiles, pour une longueur de 15 toiles. Soit une voile de 12 mètres de large sur 18 mètres de haut, pour un bateau sans quille dont le tirant d'eau n'excédait pas 75 centimètres. Le voiturier devait utiliser sa voile avec précaution car un coup de vent de travers pouvait facilement renverser son bateau.

Selon un mémoire de 1785 les premiers bateaux d'un train " portent chacun un mât, mais moins haut par gradation que le premier afin que les voiles reçoivent toutes les vents proportionnellement ${ }^{35}$ ". Ainsi, le chaland maté reçoit assez de vent dans les voiles pour mener le train de bateaux sans être rattrapé. Cette gradation de la voilure dans les trains de bateaux se voit dans certains inventaires après décès où la taille des voiles varie selon la position des bateaux : dans un inventaire de $1779^{36}$, le chaland mâté a une voile de 11 toiles, le tirot une voile de 10, le soubre une voile de 9 , le quatrième bateau une voile de 8 . Dans d'autres procès-verbaux, cette gradation apparaît dans le poids des cordages destinés aux bateaux.

Quand le vent n'était pas favorable, les voituriers devaient haler leurs bateaux à la corde; 8 procès-verbaux sur 231 signalent le halage. Pour cela l'équipage suffisait, soit deux ou trois personnes par bateau. Mais cette méthode n'était qu'un pis-aller, car le voyage était beaucoup plus lent qu'à la voile. De plus, les chemins de halage étaient souvent inutilisables lors des crues. Sur la haute Loire où les voituriers ne bénéficiaient plus des vents d'ouest, le halage était beaucoup plus fréquent qu'en Anjou, alors

33. Pourtant une sapine matée pour remonter le courant est signalée dans un procèsverbal : " le second de leurs bateaux qui est une sapine mattée appellée le tirot " (Arch. dép. de Maine-et-Loire, 5E 13/97, notaire Legeay des Rosiers, le 8 novembre 1785).

34. Arch. dép. de Maine-et-Loire, 5E 13/443, notaire Guillemet; 5E 13/310, Cornilleau; 5E 90/601, Fruslon : inventaires des 5 janvier 1752, 20 octobre 1790 et 28 août 1758.

35. Extrait d'un "mémoire concernant la navigation des rivières sur lesquelles les entrepreneurs de la voiture du sel font le fournissement des greniers de grande gabelle " (Archives Nationales, G1/97) publié sous le titre : « La navigation sur la Loire et ses affluents vers 1785 ", dans Annales de Bretagne 36, 1, 1924-1925, pages 76-95. Aussi publié dans l'Anjou historique de janvier 1927.

36. Arch. dép. de Maine-et-Loire, 5E 13/300, notaire Gallais de Saint-Clément-des-Levées, inventaire Pierre Chaslot et Etiennette Taugourdeau du 21 juillet 1779. 
même que la force du courant nécessitait plus de personnel pour tirer les bateaux.

Pour la descente du fleuve, les bateaux n'étaient pas attachés à la file comme pour la remontée, mais deux par deux et bord à bord, afin de former ce qu'on appelait un coublage ou couplage. Un des bateaux était avancé de quelques mètres par rapport à l'autre. Les bateaux étant portés par le courant, les pautres ou gouvernails étaient inefficaces. Les mariniers devaient donc prendre appui sur le fond du fleuve pour se diriger. Ils utilisaient pour cela de solides perches de chêne appelées bâtons de quartier. Les mariniers coinçaient une extrémité des bâtons, nommée varveau, dans un arronçoir, c'est-à-dire un crénelage de bois situé sur les flancs du bateau à l'avant et à l'arrière. Ils appuyaient l'autre extrémité sur le fond du fleuve. Le bateau pesait sur les bâtons et basculait du côté opposé. Les bâtons de quartier permettaient aussi d'immobiliser un bateau dans le courant, en mettant un bâton de chaque côté. Les bâtons étaient enlicanés, c'est-à-dire dotés d'une corde attachée au bateau afin de les récupérer. Comme ils cassaient souvent, les voituriers en embarquaient de grandes quantités. Dans les inventaires après décès on trouve généralement une quinzaine de bâtons par bateau ${ }^{37}$. En théorie, les voituriers devaient récupérer les bouts de bâtons de quartiers cassés qui restaient enfoncés dans le sable, mais certains ne prenaient pas le temps de le faire, ce qui provoquait de nombreux accidents.

Figure 3 - Les bâtons de quartier

(Source : extrait d'une carte postale du début du XXe siècle)

Le document représente le passage du pont des Rosiers par un chaland On voit les bâtons de quartier posés à l'avant du bateau

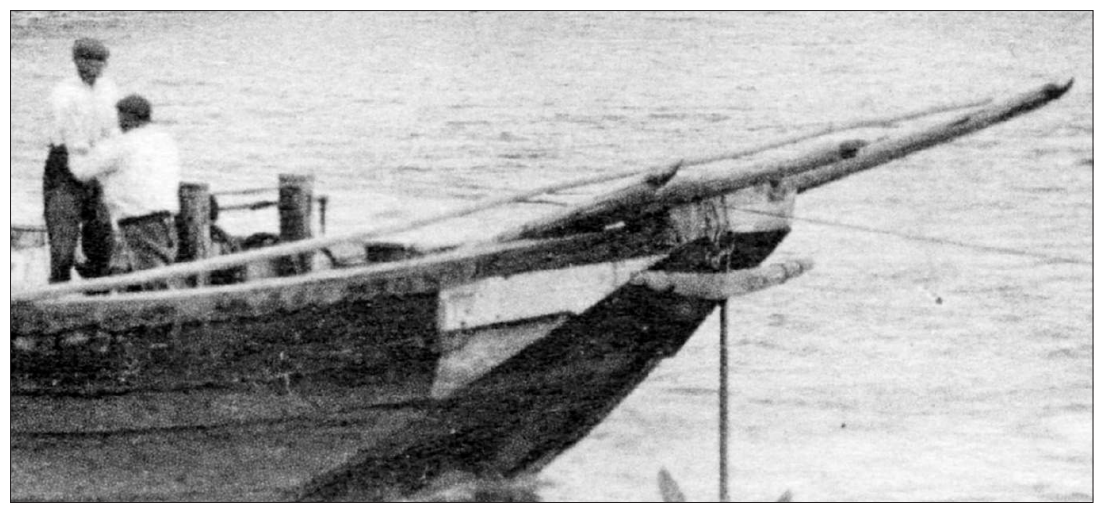

37. Un inventaire de 1732 comprend 110 bâtons pour six bateaux : chaland, tirot, deux soubres et deux sapines (Arch. dép. de Maine-et-Loire, 5E 55/233, notaire Roulleau de Gennes, inventaire Marie Besnard veuve Mathieu Bonnet des Ponts-de-Cé, 24 octobre 1732). En 1790, un autre inventaire comporte 29 bâtons dont 13 enlicanés pour deux bateaux, et en 1771, dans un dernier inventaire, on trouve 17 bâtons dont sept enlicanés pour un seul bateau. 
Cette utilisation des bâtons de quartier était une manœuvre brutale et très délicate ${ }^{38}$. Délicate, car elle demandait à la fois force et adresse (selon Abel Poitrineau les bâtons de quartier pesaient plus de $50 \mathrm{~kg}^{39}$ ). Brutale, parce que les forces en jeu étaient énormes. Malheur au marinier qui se coinçait un doigt entre le bâton et l'arronçoir.

\section{Combien de bateaux par voiturier? Combien de mariniers pour chaque bateau?}

Les procès-verbaux nous donnent un aperçu du nombre de bateaux naviguant de concert. La majorité des voituriers disposaient de plusieurs bateaux, mais les trains de plus de six étaient rares. Dans les procès-verbaux le nombre de bateaux des trains à la remontée correspond approximativement au nombre de bateaux possédés par les voituriers ${ }^{40}$. Par contre, le nombre de bateaux des voituriers se dirigeant vers l'aval doit être considéré avec plus de précautions : les trains de trois bateaux ou plus étant divisés pour les faire redescendre par deux, les voituriers négligeaient souvent d'indiquer le nombre total de leurs bateaux, et ne signalaient que ceux des couplages endommagés. Résultat : alors que nous avons à peu près le même nombre de procès-verbaux concernant des bateaux se dirigeant vers l'amont et vers l'aval, les trois-quarts des procès-verbaux signalant plus de trois bateaux correspondent à des voyages vers l'amont (50 cas sur 66) (Tableau 1).

Onze inventaires après décès nous renseignent aussi sur le nombre de bateaux possédés par des voituriers angevins. Là encore, la majorité des voituriers par eau possédaient plusieurs bateaux, mais seuls deux en avaient six ou sept (Tableau 2).

Les trains de bateaux transportant du sel étaient de plus grande taille que la moyenne car le voiturage du sel, produit très surveillé car soumis à la gabelle, faisait l'objet de contrats sur de grosses quantités, passés avec de riches voituriers disposant de nombreux bateaux. À travers divers documents - procès-verbaux de réception de sel dans un grenier, contrats entre voituriers et mariniers, procès-verbaux d'avaries... - on connaît cinq trains de bateaux pour le sel, dont trois trains de huit bateaux, un de sept bateaux et un de quatre bateaux ${ }^{41}$.

38. François Beaudouin a bien décrit cette manœuvre dans « La marine de Loire et son chaland ", op. cit.

39. PoitrinEAu, Abel, "La Loire Marchande, les trafics d'antan ", dans Une histoire de la Loire, Paris, Ramsay, 1986, p. 105.

40. Dans trois cas seulement le voiturier est remplacé par un "facteur ", marinier faisant office d'homme de confiance. Et les inventaires après décès de biens appartenant à des mariniers ne signalent qu'un train de bateaux à chaque fois (11 inventaires étudiés, voir plus loin).

41. Arch. dép. de Maine-et-Loire, 5E 96/1, notaire Gaudin d'Ingrandes, en 1710 réception de sel au grenier d'Ingrandes. 5E 13/281, notaire Guittoneau des Rosiers, marchés de voiturage (entre voituriers et mariniers) des 9 novembre 1786 et 17 février 1787. C 85, Débâcle des glaces dans la subdélégation de Saumur, bateaux de Claude Picard. 5E 108/8, notaire Gazeau de Saint-Florent-le-Vieil, avarie du 11 mars 1790. 
Les bateaux de Loire nécessitaient un équipage relativement réduit au vu de leurs capacités de charges. Selon un mémoire rédigé vers $1786{ }^{42}$, l'équipage des bateaux de Loire était composé d'un maître et de deux hommes d'équipage. En amont d'Orléans le nombre de mariniers était multiplié par cinq ou dix, car il fallait abandonner la voile pour le halage, en raison du courant et du manque de vent. L'équipage complet apparaît dans 67 procès-verbaux d'avaries (Tableau 3).

Tableau 1 - Nombre de bateaux possédés par les voituriers angevins d'après les procès-verbaux

\begin{tabular}{|c|c|c|}
\hline Nombre de bateaux & $\begin{array}{c}\text { Nombre de procès-verbaux } \\
\text { Remontée }\end{array}$ & $\begin{array}{c}\text { Nombre de procès-verbaux } \\
\text { Descente }\end{array}$ \\
\hline 1 & 20 & 21 \\
2 & 26 & 32 \\
3 & 18 & 4 \\
4 & 12 & 7 \\
5 & 5 & 1 \\
6 & 13 & 2 \\
7 & 2 & 1 \\
8 & 1 & \\
9 & & 1 \\
12 & 16 & 18 \\
Plusieurs & & 17 \\
Nombre inconnu & 8 & \\
\hline
\end{tabular}

Tableau 2 - Nombre de bateaux possédés par les voituriers angevins d'après les inventaires après décès

\begin{tabular}{|l|l|l|l|l|l|l|}
\hline $\begin{array}{l}\text { Nombre de bateaux } \\
\text { par voiturier }\end{array}$ & 1 & 2 & 3 & 4 & 6 & 7 \\
\hline Nombre d'inventaires & 1 & 5 & 2 & 1 & 1 & 1 \\
\hline
\end{tabular}

Tableau 3 - Équipage moyen par bateau à partir de 67 procès-verbaux

\begin{tabular}{|c|c|c|c|c|c|c|c|}
\hline Nombre de bateaux par train & 1 & 2 & 3 & 4 & 5 & 6 & 7 \\
\hline Nombre de trains & 11 & 25 & 8 & 9 & 1 & 6 & 7 \\
\hline Total des équipages ${ }^{45}$ & 29 & 82 & 57 & 46 & 7 & 57 & 9 \\
\hline Moyenne par bateau & 2,63 & 1,64 & 2,37 & 1,27 & 1,4 & 1,58 & 1,29 \\
\hline
\end{tabular}

42. Rapport de Soland, commissaire des classes de la marine à Angers en 1786, adressé aux inspecteurs des classes de Bretagne. Arch. dép. de Maine-et-Loire, C 22, navigation en Loire. Publié sous le titre "La navigation de l'Anjou en 1786 " dans Mémoires de la société des sciences et arts d'Angers, 1908, 5e série, T XI, p. 205-214.

43. Dans 34 procès-verbaux, le voiturier possédait plusieurs bateaux, sans que soit précisé leur nombre. Les 18 procès-verbaux concernant des voyages vers l'aval ont été pris en compte dans la ligne du dessous et font partie des 66 cas relevés où les voituriers avaient plusieurs bateaux.

44. Il faut ajouter les toues de bord, au moins une pour deux bateaux.

45. Ce total comprend le ou les voituriers ou leurs représentants ainsi que leurs familles, mais sans les mariniers recrutés ponctuellement pour passer les Ponts-de-Cé. 
Les familles des voituriers sont assez peu représentées dans les équipages. Six voituriers déclarent voyager avec leur fils, et trois avec leur femme. La présence des femmes est rare, mais on peut remarquer trois équipages majoritairement féminins entre 1765 et $1768^{46}$.

\section{Des temps de trajet irréguliers}

Selon des ingénieurs des Ponts et Chaussées cités par Abel Poitrineau, en 1834 les chalands mettaient entre huit jours et deux mois pour remonter le fleuve de Nantes à Orléans ${ }^{47}$. Cet écart donne une idée des incertitudes de la navigation, souvent contrariée par les basses eaux, par les grandes eaux, par le vent dans un sens ou dans l'autre, ou par l'absence de vent. Le manque de vent était le principal problème des voituriers car le vent d'ouest conditionnait la remontée. En 1860, selon un ingénieur des Ponts et Chaussées,

" nul entrepreneur de transport ne peut assurer la livraison à époque fixe des matières qu'il se charge de voiturer. De 1855 à 1859 par exemple, la navigation a été entravée chaque année (moyennes quinquenales) par les vents contraires 202 jours, par les glaces pendant 9 jours, par les brouillard pendant 8 jours ${ }^{48}$ ".

Le tableau 4, daté de 1864, confirme l'importance des vents, mais montre aussi que les basses eaux faisaient aussi obstacle à la navigation, en particulier en amont de Tours.

46. En 1765, Urbaine Lagarette épouse de Louis Dubé voiturier par eau, et Françoise Dubé épouse de Michel Salin tonnelier absent. En 1766 un équipage entièrement féminin : Renée Bonnaventure femme de Michel Pinson, Magdelaine Gaudais fille majeure, et Rose Delumeau veuve de Christophe Daburon. En 1768 Magdelaine Niollet fille majeure associée à René Boisnard, et Madelaine Gaudin. Toutes ces voiturières résident à Angers. Nous ignorons la raison de cette concentration dans l'espace et dans le temps (1765 à 1768). Est-ce encore une coïncidence? Deux de ces trois procès-verbaux concernent des collisions provoquées par d'autres voituriers heurtant leurs bateaux par l'arrière. Pourtant les collisions ne représentent que $5 \%$ du total des avaries. Peut-être ces femmes ont-elle été victimes du "machisme " de certains voituriers essayant de leur faire peur en naviguant très près d'elles, et perdant le contrôle de leurs bateaux? Il est peu probable que ces accidents soient provoqués volontairement, car les voituriers fautifs en supportent les conséquences financières. (Arch. dép. de Maine-et-Loire, 5E 53/59, notaire Leduc de Chalonnes, procès-verbal du $1^{\text {er }}$ mars 1765. 5E 19/8, Dertron de Savennières, procès-verbal du 4 octobre 1766. 5E 96/157, Mercier de Montrelais, procès-verbal du 29 mars 1768).

47. PoItrineAu, Abel, « L'économie du transport fluvial. Une esquisse ", Revue historique, $\mathrm{n}^{\circ} 577$, janvier-mars 1991, p. 115.

48. Rapport de l'ingénieur Collin, des Ponts et Chaussées, 1860, dans Aspects de l'histoire sociale et politique du Département du Loire-et-Cher de 1848 à 1914 de Georges Dupeux, cité par MARCILHACY Christiane, "Les mariniers et la marine de la Loire vus du port de Châteauneuf-sur-Loire ", Arts et Traditions populaires, 1960, p. 56. 

Tableau 4 - Nombre moyen de jour de chômage
ou de difficultés en $1864^{49}$

\begin{tabular}{|l|c|c|c|c|c|}
\hline \multirow{2}{*}{$\begin{array}{l}\text { Sections partielles de } \\
\text { la Loire entre Briare } \\
\text { et Nantes }\end{array}$} & $\begin{array}{c}\text { Les basses } \\
\text { eaux } \\
\text { (chevalage) }\end{array}$ & Les crues & Les glaces & Les brouillard & $\begin{array}{c}\text { L'absence de } \\
\text { vents favora- } \\
\text { bles à la } \\
\text { remontée }\end{array}$ \\
\cline { 2 - 6 } & 270 jours & 1 jour & 28 jours & 9 jours & 229 jours \\
\hline $\begin{array}{l}1^{\text {re }} \text { section de Briare } \\
\text { à Combleux }\end{array}$ & 180 & 1 & 20 & 5 & 225 \\
\hline $\begin{array}{l}2^{\mathrm{e}} \text { section de Combleux à } \\
\text { Tours (confluent du Cher) }\end{array}$ & 121 & 0 & 19 & 0 & 190 \\
\hline $\begin{array}{l}3^{\mathrm{e}} \text { section de Tours au } \\
\text { confluent de la Vienne }\end{array}$ & 110 & 0 & 19 & 0 & 194 \\
\hline $\begin{array}{l}4^{\mathrm{e}} \text { section de la Vienne } \\
\text { à la Maine }\end{array}$ & 100 & 0 & 17 & 0 & 150 \\
\hline $\begin{array}{l}5^{\mathrm{e}} \text { section de la Maine } \\
\text { à Nantes }\end{array}$ & & & & & \\
\hline
\end{tabular}

Les crues trop fortes pouvaient rendre la navigation impossible car trop dangereuse. Les voituriers risquaient de ne pas trouver terre avec leurs bâtons de quartiers alors même que le courant les entraînait avec rapidité. D'autre part, le halage devenait impossible si la crue atteignait deux ou trois pieds au-dessus du rivage ${ }^{50}$ et les arches des ponts, souvent trop basses, n'étaient plus franchissables ce qui interrompait la navigation. Enfin certains passages particulièrement difficiles nécessitaient un bon vent pour les franchir, ainsi la région appelée La Courbe en amont des Ponts-de-Cé ${ }^{51}$. Arrêtés par les vents, par les glaces, par une crue ou au contraire par une forte baisse du niveau des eaux, les voituriers pouvaient rester bloqués plusieurs semaines et même parfois plusieurs mois avant de pouvoir reprendre leur voyage ${ }^{52}$.

49. Arch. dép. de Loire-Atlantique, 592-S-1, relevé par Amélie Dubois-Richir dans La Loire au XIX $X^{e}$ siècle : le fleuve et ses riverains de Saumur à Bouchemaine, thèse d'histoire, Angers, 2001, p. 305 bis.

50. Dans plusieurs procès-verbaux, les voituriers affirment pour leur défense qu' on ne peut faire remonter le bateau par le tirage de la halée les eaux etant debordées de plus de trois pieds sur les isles islots et sur les chantiers".

51. " après avoir acquitté au bureau de cette ville, il se mit promptement au comble du pont et hissa ses voilles pour profiter du bon vent qui soufflait lors, sachant par expériance qu'une heure de retardement, si le vent qui soufflait lors vennait à changer, ou a cesser, il seroit peut estre de longtemps impossible de remonter une certaine étendue de la rivière nommée la courbe par les difficultés sans nombre qui sy rencontrent tant à cause des greves et des isles, qu'accause quil ny a quun certain petit espace d'eau qui puisse porter les bateaux. Le vent qui soufflait allors etant le seul qui put le mettre en estat de surmonter touttes les difficultés, il avait donc disposé ses voilles et ses agrets pour profiter de ce vent favorable " (Arch. dép. de Maine-et-Loire, 5E 90/625, notaire Fruslon des Ponts-de-Cé, procès-verbal du 6 octobre 1786).

52. Un voiturier parti de Nantes 16 ou 17 jours plus tôt " seroit près d'arriver à Orléans sans les grandes eaux ", alors qu'il est bloqué aux Ponts-de-Cé, à un ou deux jours de 
Les retards pouvant être importants, les voituriers demandaient parfois aux notaires de dresser des procès-verbaux pour se justifier auprès de leurs clients. D'autres voituriers, des riverains ou le notaire lui-même, étaient priés de témoigner de l'impossibilité matérielle pour les voituriers de reprendre leur voyage. Nous disposons de vingt-sept de ces documents appelés procès-verbaux de retard de livraison ${ }^{53}$. Dans ces procès-verbaux les principales causes de retard sont les éléments naturels (crues, vents, glaces) et les péages (Tableau 5). Ces procès-verbaux étaient souvent liés à l'établissement d'un " jour nommé " (13 cas sur 27), c'est-à-dire qu'une date et donc une durée étaient fixées dans le contrat de voiturage pour la livraison des marchandises. Le délai maximum accordé aux voituriers pour faire le voyage de Nantes à Orléans était de 18 à 25 jours (souvent 20 jours). En sens inverse, la descente étant plus rapide que la remontée, le délai était de 11 à 15 jours $^{54}$. Les temps de trajets définis par les jours nommés laissaient probablement une petite marge aux voituriers dans des conditions normales de navigation. Les procès-verbaux d'avaries indiquent rarement des temps de trajet, mais l'un d'eux signale un départ d'Orléans le 26 janvier 1752 et une arrivée à Ingrandes le 3 février, ce qui fait un voyage de huit jours, donc en principe neuf jours jusqu'à Nantes. On pourrait s'attendre à ce que les voituriers en retard soient pénalisés sur le prix de leur "voiture ", mais concrètement les jours nommés avaient surtout pour utilité d'inciter les voituriers à aller le plus vite possible sans chercher à compléter leur chargement en chemin. Les marchands savaient bien que les temps de trajet sur la Loire étaient aléatoires. En montrant dans un procèsverbal, avec des témoins, que le retard était indépendant de sa volonté et causé par une crue ou une défaillance du vent, le voiturier pouvait obtenir le report du jour nommé à une date fixée à l'avance ${ }^{55}$ ou indéterminée ${ }^{56}$.

trajet de son point de départ. À la fin janvier 1789, beaucoup de bateaux étaient bloqués par les glaces depuis novembre 1788. Un bateau bloqué à Sainte-Gemmes-sur-Loire à la fin janvier 1789 était même parti le 17 octobre de Nantes (Arch. dép. de Maine-et-Loire, $5 \mathrm{E} 8 / 65$, notaire Brevet d'Angers, procès-verbal du 26 janvier 1789).

53. 26 nous renseignent sur l'origine et la destination des marchandises : 13 voituriers remontaient la Loire et 13 la descendaient. L'essentiel du trafic se faisait entre Nantes et Orléans. La durée du voyage est précisée dans sept cas : cinq à la remontée et deux à la descente.

54. C'était une des raisons du coût plus élevé du transport à la remontée. D'après des enquêtes effectuées à la Révolution dans le cadre de la fixation du maximum des prix, le coût des transports sur la Loire était 1,5 à 2,5 fois plus élevé à la remontée qu'à la descente (REMOND, André, Les prix des transports marchands de la révolution au Premier Empire, 1956). Une autre enquête de 1817 évalue le fret à 0,13 franc par millier et par lieue en moyenne à la descente et à 0,38 franc à la remontée (PoITRINEAU, Abel, "L'économie du transport fluvial. Une esquisse ", Revue historique, ${ }^{\circ} 577$, janvier-mars 1991, p. 116).

55. «Son jour nommé ne pourra commencer que lundy prochain vu le temps contraire " (Arch. dép. de Maine-et-Loire, 5E 96/16, notaire Gaudin d'Ingrandes, procès-verbal du 27 décembre 1743).

56. «Protestant que le jour nommé ne poura courir que les eaux ne soient retirées et le vent agrez et favorable ce qui ne paroist pas estre de plus de dix a douze jours " (Arch. dép. de Maine-et-Loire, 5E 96/16, procès-verbal du 8 février 1741). 
Si le retard se prolongeait, certains voituriers demandaient même " récompense et dommages et intérêts " en compensation de la perte de temps et du coût de la nourriture de leurs hommes ${ }^{57}$. Mais nous ignorons s'ils obtenaient satisfaction...

Tableau 5 - Les causes de retard dans les procès-verbaux

\begin{tabular}{|c|c|c|c|c|c|}
\hline Basses eaux & Glaces & Vent & $\begin{array}{c}\text { Vent et crues } \\
\text { conjugués }\end{array}$ & Crues & $\begin{array}{c}\text { Péages et } \\
\text { erreurs des } \\
\text { expéditeurs }\end{array}$ \\
\hline 1 & 3 & 5 & 4 & 7 & 8 \\
\hline
\end{tabular}

Aux conditions naturelles s'ajoutaient les péages de toute sortes. Les voituriers devaient faire contrôler leurs " lettres de voiture " (contrats de voiturage) par les commis chargés de la perception des droits, et souvent subissaient une visite des bateaux. À Ingrandes, pour l'impôt de la cloison d'Angers, on pesait certaines marchandises à bord d'un bateau prévu à cet effet et on perçait des trous dans les tonneaux pour en vérifier le contenu. Lorsque de nombreux voituriers arrivaient en même temps, portés par la même crue de la Loire, ils devaient patienter longtemps avant d'être contrôlés. Dans un procès-verbal de 1782, un voiturier se plaint d'avoir attendu deux jours à Saumur et trois jours aux Ponts-de-Cé pour pouvoir faire viser ses lettres de voiture ${ }^{58}$. Plus grave, des voituriers voyaient faiblir la crue qui les avait amenés, ou perdaient le bénéfice du vent. Un jour d'attente à la douane pouvait entraîner plusieurs semaines de retard ${ }^{59}$. D'autre part, la moitié des retards liés aux péages étaient dus à l'insuffisance des fonds confiés par les marchands aux voituriers pour payer les taxes. À la décharge des marchands, il était difficile au XvIII ${ }^{\mathrm{e}}$ siècle de prévoir exactement les sommes à payer aux différents péages entre Nantes à Orléans. Ces péages étaient nombreux, portaient sur des marchandises différentes, à des taux variés et leurs règlements souvent anciens et inadaptés étaient interprétés de diverses manières. Les abus et les contestations étaient bien

57. Arch. dép. de Maine-et-Loire, 5E 90/575, notaire Dubourg des Ponts-de-Cé, procèsverbal du 7 novembre 1732 .

58. Arch. dép. de Maine-et-Loire, 5E 90/658, notaire Foucher des Ponts-de-Cé, procèsverbal du 18 mars 1782.

59. En Anjou, les deux principaux péages étaient la Cloison d'Angers et le Trépas de Loire. Ils furent supprimés à la Révolution. Le péage de la Cloison apparaît pour la première fois dans un texte en 1372 pendant la Guerre de Cent Ans pour payer la fortification de la ville d'Angers. Le Trépas de Loire avait été levé pour la première fois en 1369 à la demande de Duguesclin pour payer le départ d'une troupe anglaise retranchée à l'abbaye de Saint-Maur-sur-Loire. Au XVIII ${ }^{\mathrm{e}}$ siècle, l'impôt de la Cloison se levait à Angers, à Bouchemaine, aux Ponts-de-Cé et à Ingrandes. Le Trépas de Loire était perçu sur les marchandises entre Candes-Saint-Martin et Ancenis. Les voituriers obtenaient au péage un " acquet " ou sauf-conduit qui permettait de franchir les autres lieux de perception. MERLET, Monique, Le péage de la cloison d'Angers ( $X V^{e}-X V I I I^{e}$ siècle), contribution à l'étude du trafic de la Loire à la fin de l'Ancien Régime, thèse de $3^{\mathrm{e}}$ cycle, École nationale des chartes, p. 6 sqq. 
sûr fréquents. Ces douanes intérieures furent réformées à la Révolution, ce qui réduisit un peu les temps de trajet $^{60}$.

\section{Les naufrages en Loire et leurs conséquences}

\section{Les principales causes de naufrage: les tempêtes, les obstacles immergés et les pertes de contrôle}

Les informations tirées des procès-verbaux sur les causes d'avaries sont à prendre avec précaution car ces documents présentent le point de vue des voituriers. Les éléments naturels, la maladresse et la négligence d'autres mariniers ou la fatalité étaient toujours incriminés. Sur un total de 204 procès-verbaux d'avaries, 196 concernent des bateaux, 4 des trains de bois et 4 les marchandises seulement. Voici la répartition des causes d'avaries.

\section{Tableau 6}

\begin{tabular}{|c|c|c|c|}
\hline Causes & & Précisions & \\
\hline Intempéries & 51 & $\begin{array}{l}\text { - Coups de vent, tempêtes, tourbillons } \\
\text { - Glaces }\end{array}$ & $\begin{array}{l}40 \\
11\end{array}$ \\
\hline $\begin{array}{l}\text { Pertes de contrôle } \\
\text { et collisions }\end{array}$ & 27 & $\begin{array}{l}\text { - Échouages sur des îles, rochers, bancs de sable } \\
\text { - Moulins flottants } \\
\text { - Heurts dans un train ( } 2 \text { cas) ou dans un couplage ( } 1 \text { cas) } \\
\text { - Heurts entre trains de bateaux }\end{array}$ & $\begin{array}{c}11 \\
3 \\
3 \\
10\end{array}$ \\
\hline Obstacles immergés & 93 & $\begin{array}{l}\text { - Bâtons de quartier } \\
\text { - Branches, troncs d'arbres, bois charriés } \\
\text { - Rochers submergés } \\
\text { - Bois flottants } \\
\text { - Pieux indéterminés } \\
\text { - "Drajon " ou pieu de pont } \\
\text {-Épave de bateau }\end{array}$ & $\begin{array}{c}36 \\
30 \\
12 \\
6 \\
7 \\
1 \\
1\end{array}$ \\
\hline Passage de ponts & 21 & - Courant et tourbillons projetant sur les pilles, pieux. & \\
\hline Divers & 3 & $\begin{array}{l}\text { - Échouage par négligence }{ }^{61}, \text { chute de talus, } \\
\text { casse d'une ferrure de bateau liant les planches du fond }\end{array}$ & \\
\hline
\end{tabular}

Les principales causes de naufrages étaient les obstacles immergés, les coups de vent et tempêtes, les pertes de contrôle des bateaux et les glaces. De nombreuses avaries se produisaient aussi lors des passages de ponts. Les obstacles immergés étaient tenus pour responsables de près de la moitié des avaries étudiées ici (93 cas relevés), et parmi ces obstacles domi-

60. En octobre 1790, des voituriers bloqués depuis deux semaines faute d'argent profitèrent de la suppression de ces douanes pour trouver une échappatoire : "Ce ne fut qu'hier quils en obtirent permission [de partir] du directeur du bureau des traites d'après les nouvelles quil leur dit avoir recues du decret nouvellement rendu par l'assemblée nationale portant qu'au premier décembre prochain les bureaux des traites dans l'intérieur du royaume seront transférés sur les frontières " (Arch. dép. de Maine-et-Loire, 5E 42/79, notaire Challopin de Saumur, procès-verbal du 21 octobre complété le 4 novembre 1790 ).

61. Le niveau de l'eau s'est abaissé et un bateau chargé d'huîtres s'est retrouvé à sec. 
naient les bâtons de quartier, pieux, troncs d'arbres et rochers. Les solides bâtons de quartier utilisés pour la conduite des bateaux cassaient souvent à cause des forces énormes auxquelles ils étaient soumis. Laissés par des voituriers n'ayant pas réussi à les récupérer ou peut-être pas tenté - cela demandait du temps - ils représentaient un grand danger pour la navigation en Loire. En 1809, un arrêté préfectoral obligea les voituriers à graver leur nom sur leurs bâtons et à en déclarer le nombre ${ }^{62}$, ce qui devait permettre aux victimes d'avaries de se retourner contre les anciens propriétaires des bâtons. Bien entendu, les voituriers déclaraient que les objets à l'origine de leurs accidents étaient invisibles depuis la surface, " ne faisant aucuns boidre ny ligne de mouvement d'eau ${ }^{63}$ " et que les rochers étaient " sans balise ni remarque ". Si l'obstacle était invisible, la responsabilité du voiturier était amoindrie. Des baliseurs enlevaient une partie des débris encombrant le chenal et signalaient avec des perches ou balises les obstacles trop importants. Certains lieux nécessitaient un balisage régulier. Sur douze avaries causées par des rochers, trois surviennent face à l'église de Bessé, près de Gennes, dont deux à un mois d'intervalle en octobre et novembre 1774 : à l'évidence, cette année-là, le balisage avait été mal fait en cet endroit. La "Compagnie des marchands fréquentant les rivières de Loire et rivières y affluentes " était chargée du balisage et du nettoyage du fleuve, mais son travail laissait à désirer ${ }^{64}$. L'intendant de la généralité de Tours signale en 1697 qu' " on se plaint en Anjou des abus qui se commettent dans le balisage de la rivière de Loire, notamment au-dessus des Pontsde-Cé, où on ne travaille que fort rarement ${ }^{65}$ ". En 1772, la Compagnie fut supprimée et on confia le balisage à des entrepreneurs, puis en 1783 il passa directement sous la responsabilité des ingénieurs de l'administration des Turcies et Levées. Un règlement minutieux fut édicté mais mal appliqué. Malgré une amélioration, les naufrages restèrent fréquents faute d'un bon balisage. En 1786 à Saumur un voiturier affirmait : "Le balisage n'a point été fait cette année sur ladite rivière de Loire ${ }^{66}$. " Selon l'Inspecteur Général des Turcies et Levées de la généralité de Tours, il y eut trente-sept bateaux naufragés ou avariés de 1784 à 1787. Il déclara pour sa défense :

"Quand il y aurait dix, vingt mille mariniers, cinq cents ou mille bateaux de balisage [...] quand cette armée veillerait jours et nuits à baliser, elle

62. Jeanne et Camille FRAYSSE, Loire angevine et Maine, Cholet, 1974, p. 87.

63. Le mot " boidre ", très courant, désigne un remous en surface.

64. La Compagnie employait un seul équipage de mariniers pour le balisage entre Roanne et la Bretagne (sept équipages créés après 1772). Tous les bois coupés sur les rives pour le chemin de halage étaient vendus à leur profit, ce qui entraînait des abus et de nombreux procès. François Dumas, La Généralité de Tours au XVIII siècle (1766-1783), extrait des mémoires de la société archéologique de Touraine, t. 39, Paris, 1894, p. $250,251$.

65. « Mémoire sur l'Anjou » de 1697 par Miromesnil, intendant de la Généralité de Tours. Publié par MARCHEGAY dans Archives d'Anjou, Angers, 1843, tome 1.

66. Arch. dép. de Maine-et-Loire, 5E 42/78, notaire Chaloppin de Saumur, procès-verbal du 30 octobre 1786 . 
n'empêcherait pas quelques naufrages dans cette étendue, causés ou par des souches, ou par des arbres, ou par des bâtons cassés. Cette armée n'empêcherait pas non plus les naufrages causés par les tempêtes, par les inondations, par les glaces, par les friponneries des patrons, des mariniers, et que tant qu'on naviguera sur les rivières il y aura des naufrages ${ }^{67}$."

Quarante avaries étaient attribuées à des tempêtes et au vent. La déclaration du Roi d'avril 1703 qui réglementait les conditions de navigation faisait défense aux voituriers " de se mettre en chemin en tems de gros vent et tempête, à peine de demeurer responsables de la perte des marchandises et des dommages et intérêts des marchands ${ }^{68}$ ». Autant dire que dans les procès-verbaux rédigés à la demande des voituriers, ces derniers étaient souvent victimes de coups de vent soudains et imprévisibles qui se prenaient dans les voiles et chaviraient les bateaux sans qu'ils aient le temps de faire quoi que ce soit ${ }^{69}$. Certains reconnaissaient qu'ils avaient repris leur voyage malgré un temps menaçant, et que leurs bateaux avaient été submergés ou disloqués par les vagues et la pluie ${ }^{70}$. Mais ils pouvaient alléguer pour leur défense leur volonté de satisfaire les marchands en accélérant leur voyage. Quand une tempête s'annonçait il était prudent de trouver une gare à l'abri du vent et de la houle, dans un bras latéral de la Loire par exemple. Les grandes tempêtes pouvaient faire de multiples victimes. Celle de la nuit du 14 au 15 mars 1751 fut, selon un ordonnance du Présidial d'Angers, " si violente que de mémoire d'hommes on n'a pas essuyé un accident si funeste ${ }^{71}$ ". Cinq bateaux et une toue coulèrent cette nuit là aux Ponts-de-Cé ${ }^{72}$, et quatre autres bateaux au port de Saint-Rémy-la-Varenne ${ }^{73}$. Le 24 janvier 1724, un tourbillon de vent provoqua deux naufrages à Savennières, et le 4 décembre 1741, un notaire dénombrait au total trois naufrages le même jour dans les environs d'Ingrandes.

La perte de contrôle des bateaux et les collisions étaient une autre cause fréquente d'avaries. On peut facilement soupçonner les mariniers de cacher leurs responsabilités car les facteurs étaient multiples : le vent, les écueils visibles, le courant, les méthodes de navigation. De nombreuses

67. DumAs, François, op. cit., page 259.

68. Arch. dép. de Maine-et-Loire, C 22 navigation en Loire. Déclaration du Roi du 24 avril 1703 " pour le rétablissement et augmentation du commerce et de la navigation de la Loire, et autres Fleuves y affluans".

69. "Vent si impétueux au point de jeter le mat et mature en bas de costé du nord en travers les bords dudit bateau " (Arch. dép. de Maine-et-Loire, 5E 17/205, notaire Jeffray de la Daguenière, procès-verbal du 30 septembre 1787).

70. « Un de ses batteaux se trouveant battu par les flaux de leau et la tempeste continuant se seroit separé en deux et que les morceaux dudit batteau ainsy que la marchandise dont il etoit charge sen vont au gre de leau ". Arch. dép. de Maine-et-Loire, 5E 16/21, notaire Chaillou de Saint-Mathurin, procès-verbal du 10 mars 1759.

71. Arch. dép. de Maine-et-Loire, 1B270, Présidial d'Angers, lutte contre les fléaux.

72. Arch. dép. de Maine-et-Loire, 5E 90/597, notaire Couau des Ponts-de-Cé, procès-verbal du 15 mars 1751.

73. Registres paroissiaux de Saint-Rémy-la-Varenne, GG 1-30, d'après Célestin Port, Supplément à la série E. 
avaries étaient dues à des échouages sur des rochers ou des îles et quelques-unes ou à des heurts avec des moulins-bateaux ${ }^{74}$. Dans 4 cas sur 11 , les bateaux arrêtés dans leur élan par une grève de sable ou une île étaient fracassés par les autres bateaux des trains. Les collisions entre bateaux voyageant séparément étaient fréquentes. D'autres heurts pouvaient aussi se produire entre des bateaux voyageant ensemble : en 1786 près de Sorges (en amont des Ponts-de-Cé) un tourbillon de vent fit ralentir et serpenter un train de six bateaux qui se tassèrent les uns sur les autres. Cet exemple illustre à quel point la conduite d'un train de bateau était délicate. Les voituriers devaient harmoniser la vitesse des bateaux matés, éviter les arrêts brusques, repérer le chenal de navigation qui souvent changeait d'une année à l'autre et louvoyait entre des îles et des bancs de sable, tenir compte du courant qui ne suivait pas forcément le chenal, de la direction et de la force du vent, de la force d'inertie des bateaux. Les voituriers dirigeaient leurs bateaux au milieu de ces éléments avec la pautre (ou gouvernail) du premier bateau du train (mais uniquement pour remonter le courant), avec les bâtons de quartier et enfin en modulant la surface des voiles. De nombreux procès-verbaux attribuaient les avaries à l'impossibilité de toucher terre avec les bâtons de quartier ${ }^{75}$. Il valait donc mieux rester à quai lors des grandes crues. Pour éviter un obstacle à la descente, avec un bateau seul ou avec un couplage, les voituriers avaient aussi la possibilité de jeter leurs ancres, mais parfois l'ancre n'accrochait pas, et d'autres fois les bateaux d'un couplage pouvaient se heurter ${ }^{76}$. Cette manœuvre était hasardeuse et ne se faisait qu'en cas de nécessité.

Même à l'arrêt les voituriers risquaient de faire naufrage. Les accidents dus à des tempêtes se produisaient parfois de nuit, comme six des dix-neuf accidents causés par des pieux ou des rochers : soit une tempête agitait le bateau qui heurtait les pieux, soit le niveau du fleuve baissait et le bateau reposait sur eux. Les bois portés par le courant - essentiellement des troncs d'arbres - pouvaient aussi surprendre l'équipage pendant son som-

74. Trois collisions ont lieu avec des moulins flottants, appelés aussi moulins-bateaux, moulins à bacs, à baquets, ou à chalands. Les voituriers accusent les meuniers de gêner la navigation et de désobéir aux ordonnances qui leur interdisent de mettre leurs moulins dans la voie navigable. Dans ces trois heurts avec des moulins, deux voituriers se défendent en affirmant n'avoir pu trouver terre avec leurs bâtons de quartier. Le troisième affirme que ses ancres n'ont pas accroché le fond.

75. « Ne pouvant estre les maitres de conduire leur batteau au moyen de ce quils ne pouvoient trouver la terre pour y jetter leurs bastons, ils auroient crié aux mariniers qui montaient la Loire dans un grand batteau de s'arrester un moment, de baisser leur voilles, vu qu'eux comparants ne pouvaient resister au torrant de la Loire qui dans cet endroit roulle ses eaux avec une impetuosité extraordinaire " (Arch. dép. de Maine-et-Loire, 5E 90/603, notaire Fruslon des Ponts-de-Cé, procès-verbal du 8 février 1761).

76. En 1766 à Béhuard, des voituriers sont obligés de jeter l'ancre pour éviter d'aller de biais sur une île. L'ancre prend, mais " les cordages ayant bandé ont fait frapper les deux coublages l'un contre l'autre qui a fait que le bateau dudit soulas ses debouché pendant peut estre l'espace d'une demi heure " (Arch. dép. de Maine-et-Loire, 5E 19/8, notaire Dertron de Savennières, procès-verbal du 8 juin 1766). 
meil. En janvier 1780, vers une heure du matin, une épave à la dérive percuta et coula en quelques minutes un bateau aux Ponts-de-Cé ${ }^{77}$.

La périodicité des accidents variait selon leurs causes (Tableau 7). Les accidents liés au vent et aux tempêtes étaient logiquement bien plus nombreux en hiver. Mais dans les procès-verbaux, les accidents liés à des obstacles immergés se répartissent d'une manière relativement uniforme selon les saisons, avec un maximum en novembre ce qui peut paraître surprenant car à cette époque de l'année, la Loire est habituellement haute. Les obstacles immergés sont alors moins dangereux qu'en été. Cette répartition des accidents s'explique par le fait que les bateaux étaient beaucoup moins nombreux à naviguer et donc à courir le risque d'une avarie. L'intensité de la navigation joue donc aussi sur la répartition annuelle des avaries liées au mauvais temps.

Tableau 7 - Périodicité des accidents

\begin{tabular}{|l|c|c|c|c|c|c|c|c|c|c|c|c|}
\hline Causes & janv. & fév. & mars & avril & mai & juin & juil. & août & sept. & oct. & nov. & déc. \\
\hline $\begin{array}{l}\text { Obstacles } \\
\text { Immergés }\end{array}$ & 8 & 6 & 9 & 5 & 8 & 9 & 5 & 8 & 4 & 8 & 12 & 5 \\
\hline $\begin{array}{l}\text { Vent, } \\
\text { tempêtes }\end{array}$ & 8 & 5 & 10 & 1 & 1 & 2 & 1 & 2 & 2 & 1 & 2 & 5 \\
\hline Glaces & 9 & 1 & & & & & & & & & & 1 \\
\hline
\end{tabular}

\section{Les catastrophes provoquées par les glaces}

Les glaces constituaient une sérieuse menace pour les voituriers. Pendant les grands froids, les eaux se chargeaient de glaçons soudés par paquets. À la rencontre d'un obstacle - banc de sable, île ou pont - les glaces s'accumulaient en masses énormes. C'était l'embâcle ${ }^{78}$. La pression des glaces pouvait endommager les bateaux ${ }^{79}$. Cependant, la débâcle ou dessere était encore plus dangereuse : à l'occasion d'un réchauffement ou d'une crue, les glaces se mettaient en mouvement et descendaient la Loire sous la forme d'énormes blocs, de murs de glace qui écrasaient tout sur leur passage. On parlait aussi d'empille et de dépille car ces amas de glace se formaient souvent aux ponts dont les arches plus étroites et plus basses que de nos jours laissaient un passage réduit. À plusieurs reprises des arches

77. Arch. dép. de Maine-et-Loire, 5E 90/618, notaire Fruslon des Ponts-de-Cé, procèsverbal du 20 janvier 1780 .

78. Dictionnaire de Littré, Débâcle : « Rupture subite des glaces qui, couvrant une rivière, en interrompaient le cours. " Embâcle : "Amoncellement de glaçons qui barre un cours d'eau dans une débâcle. "

79. Le 26 janvier 1789 à Sainte-Gemmes deux bateaux étaient bloqués par les glaces. Le voiturier et ses compagnons ne pouvaient jeter l'eau qui entrait car " l'eau que faisaient les bateaux gelait à l'instant même qu'elle y entrait [...] ils ont été obligés pour éviter une plus grande avarie de la couper avec des haches pour la jetter hors des bateaux " (Arch. dép. de Maine-et-Loire, 5E 8/65, notaire Brevet d'Angers, procès-verbal du 26 janvier 1789). La grande débâcle de 1789 était passée, mais les glaces étaient revenues après un " faux redoux ". 
de ponts - notamment aux Ponts-de-Cé - furent balayées par la pression des glaces et des eaux qu'elles retenaient ${ }^{80}$. Parfois le danger venait d'épaisses plaques qui se détachaient de la surface des îles et allaient éperonner les bateaux. En janvier 1767, trois bateaux furent coulés en six minutes par un bloc de glace soulevé d'une grève par une crue ${ }^{81}$. De même, en janvier 1768 , un bateau fut coulé par un glaçon " autrement en terme de marine un voisin » de 40 centimètres d'épaisseur sur 65 mètres de longueur ${ }^{82}$.

Les glaces étaient redoutées non seulement des mariniers mais aussi des riverains. Elles menaçaient les levées de la Loire en ravageant le revêtement et en augmentant la hauteur des eaux ${ }^{83}$. En janvier 1811, le préfet redoute un rupture des levées entre Angers et Saumur à cause des glaces. Il écrit au ministre de l'intérieur qu'à Saint-Maur,

"les glaces se sont amoncelées au point qu'elles forment un banc de 12 à 15 pieds d'epaisseur [3,90 à 4,80 mètres] qui porte sur le sol et produit l'effet d'un barrage, aussi les eaux sont elles à Saumur de 2 metres environ plus hautes qu'elles ne devraient être en proportion de la hauteur qu'elles ont au-dessus de l'etiage au Ponts-de-Cé ".

Il se rend alors aux Ponts-de-Cé :

" Nous y avons trouvé les glaces amoncelées à 4 et 5 mètres de hauteur au-dessus de l'eau; sur 22 arches ou travées qui existent sur le principal bras du fleuve 4 seulement sont ouvertes et laissent à l'eau un libre cours : toutes les autres sont obstruées de glacons depuis le fonds du sol jusques auprès du plancher. Si l'eau et les glaces n'étaient retenus par le barrage que je vous ai indiqué plus haut et par quelques autres semblables, je doute que le pont pût resister à cette charge enorme. Mais d'un autre coté pas un glacon ne franchit le pont ${ }^{84}$."

80. En 1684 « le 18 février, les glaces emportent quatre arches des Ponts de Cé; le 22, les glaces jointes à la hauteur des eaux emportent une longueur des Ponts de Cé avec deux moulins " (Journal de l'avocat Toisonnier.) En janvier 1789, quatre arches des Pontsde-Cé étaient de nouveau emportées. PORT, Célestin, « Les inondations dans le département de Maine-et-Loire ", Revue de l'Anjou, 1856. Repris dans Questions Angevines, 1884.

81. Arch. dép. de Maine-et-Loire, 5E 13/79, notaire Legeay des Rosiers, procès-verbal du 20 janvier 1767.

82. 15 pouces d'épaisseur et 200 pieds de longueur. Arch. dép. de Maine-et-Loire, 5E 90/653, notaire Foucher des Ponts-de-Cé, procès-verbal du 11 janvier 1768.

83. Le vicaire de Saint-Clément-des-levées écrivait en 1729 " Ce dépille arriva la première fois du jeudi au vendredi le long du costeau, porta sur le chantie [chantier] a Cunault des grands bateaux jusque sur les murailles a plus de trente pieds de hauteur, l'eau étant dans son cours ordinaire. Le depille du cote de la levée arriva le vendredy sur les neuf heures, la glace etant sur la levée, et l'eau retenue par la glace un peu en deça de la croix Thibault, étant sur le point de traverser en plusieurs endroits de plus de dix pieds de hauteur, ruina toutes les isles depuis Saumur jusqu'au dessus de St Mathurin, fit dans cette paroisse ou sur ses confins trois brèches [...] rompit et cassa nombre de bateaux. " (Registre des B.M.S. de Saint-Clément 1710-1729, cité par DeZANNEAU, Bénédicte, Les hommes et la Loire à Saint-Clément-des-Levées. 1750-1789, 1990, p. 46).

84. Arch. dép. de Maine-et-Loire, 121-S-20. Lettre du préfet au ministre de l'intérieur le 11 janvier 1811. La catastrophe fut évitée de justesse. La levée fut en partie submergée en amont de Saint-Mathurin, mais elle tint bon, ainsi que le pont Saint-Maurille. 
La Loire était plus souvent prise par les glaces que de nos jours, le pays traversant alors une période plus froide désignée sous le nom de "petit âge glaciaire ". Les procès-verbaux signalent des avaries provoquées par les glaces en 1719, 1726, 1729, 1753, 1767, 1768, 1784, et surtout en 1789, année marquée par une débâcle catastrophique ${ }^{85}$. Même si ces malheurs n'étaient pas à redouter tous les ans, les ravages provoqués par les débâcles étaient, pour les voituriers, à rapprocher de ceux subis lors de la tempête de 1751. Chaque procès-verbal d'avaries concernait souvent deux, trois ou quatre bateaux endommagés. En 1789, dans la subdélégation de Saumur, les pertes pour les voituriers étaient évaluées à environ 34000 livres, chiffre qui exclut l'essentiel des marchandises dont la valeur était en général nettement supérieure à celle des bateaux ${ }^{86}$. La catastrophe de janvier 1789 toucha aussi la rivière de Vienne où les glaces détruisirent de nombreux bateaux, ruinant les bateliers et enchérissant le prix des " voitures " par eau ${ }^{87}$.

Quand les glaces devenaient dangereuses, les voituriers essayaient de décharger les marchandises pour les mettre à l'abri. Parfois, ils les déposaient simplement sur la rive, faute de mieux, quitte à les rembarquer lorsqu'une crue survenait. Mais il n'était pas toujours possible de décharger les marchandises, faute de temps, d'argent, de personnel disponible, ou pour des raisons techniques : à Saint-Mathurin en janvier 1789 une cargaison d'ardoises ne put être déchargée car elles étaient soudées par les glaces.

\section{Les dangers liés au franchissement des ponts}

Le franchissement des ponts était un des points les plus délicats de la navigation en Loire. La force du courant ou sa mauvaise orientation sous les arches provoquaient de nombreux accidents. Le passage était rendu encore plus périlleux par des obstacles tels que des pieux de pêcheries ou des moulins-bateaux amarrés en aval des arches. Les Ponts-de-Cé étaient particulièrement redoutés car à ces inconvénients s'ajoutaient la vétusté des ponts et une mauvaise orientation du courant. Henri Chevaleau, un ancien voiturier, écrivait dans la première moitié du XIX ${ }^{\mathrm{e}}$ siècle que les Ponts-

85. Parmi les procès-verbaux étudiés, 11 relatent des avaries dues aux glaces, dont 4 pour le seul mois de janvier 1789. On dispose pour l'études des dégâts causés par les glaces en janvier 1789 de 4 procès-verbaux de naufrage, de procès-verbaux de retard et d'une enquête sur les pertes subies par les mariniers de la subdélégation de Saumur (Arch. dép. de Maine-et-Loire, C85). En 1789 la débâcle s'étala dans le temps : à SaintMathurin-sur-Loire elle se produisit dans la nuit du 18 au 19 janvier. Les 20 et 21 janvier les glaces s'accumulèrent aux Ponts-de-Cé, et le 25 janvier elles se mettaient en mouvement à l'île de Béhuard.

86. Communes de Saint-Martin-de-la-Place, Saint-Lambert-des-Levées, Saumur, Montsoreau et Villebernier. Une quarantaine de grands bateaux, 11 toues et 2 pillards étaient endommagés ou coulés.

87. Creuze-Latouche, Jacques-Antoine, Description topographique du district de Chatelleraud, département de la Vienne..., P. J. B. Guimbert, Châtellerault 1790, p. 29 (réimprimé Maxwell 1992). 
de-Cé étaient les plus craints avec ceux de Beaugency : " Ce qui est bon aux Ponts-de-Cé serait ailleurs signalé comme dangereux ${ }^{88}$. " Dans les procèsverbaux étudiés, sur 21 avaries liées aux ponts, 19 s'étaient produites aux Ponts-de-Cé, une à Saumur et une au pont de Bellepoulle (sans doute le pont de Sorges sur l'Authion). 18 des 19 avaries aux ponts de Cé s'étaient produites à la descente contre une seule à la remontée : la descente devait être la manœuvre la plus dangereuse, du moins en ce lieu.

Le passage d'un pont prenait plusieurs heures, voire une demi-journée. En 1835, des mariniers déclaraient qu'il fallait " de trois à quatre heures pour mouiller, démâter, remâter et se tirer au halage jusqu'à ce que les premiers bateaux puissent hisser les voiles ${ }^{89}$ ". Henri Chevaleau estimait lui qu'il fallait six heures pour passer un pont, et dix hommes pour descendre le mât d'un grand chaland. Pour ça, "si le train n'avait que trois ou quatre bateaux et cinq a six hommes pour équipage, ils étaient obligés à chaque pont de prendre des aides, souvent très maladroits, et que l'équipage payait très cher ${ }^{90}$ ". Des mariniers du cru louaient leurs services pour renforcer les équipages. La pratique était courante, notamment à Digoin et à la Charité-sur-Loire ${ }^{91}$. Aux Ponts-de-Cé, seuls quatre procès-verbaux sur dixneuf signalent l'embauche de mariniers supplémentaires, en nombre égal ou supérieur à celui des compagnons constituant l'équipage.

Les bateaux qui arrivaient de l'amont en couplages, c'est-à-dire attachés par deux, étaient découplés pour passer un à un sous les arches. Selon Chevaleau,

" on mouille une ancre à quatre ou cinq longueurs de bateau du pont au moyen d'une haussière, on le laisser dériver doucement sur cette ancre [...] un homme dans un bachot [petit bateau] tient à la main un grelin dont l'autre bout est fixé sur l'ancre même. Quand il veut dériver, il tire et l'ancre à demi arrachée laboure le fond en chassant. Le marinier, l'œil fixé sur la haussière, juge l'effet obtenu, et quand il veut tenir bon, il lâche l'ancre qui s'enfonce de nouveau et retient nécessairement le bateau [...] la maladresse ou une simple distraction des mariniers, la rupture de la haussière, l'ancre ne prenant pas, un accident quelconque à l'ancre [...] provoqueraient la perte de l'embarcation ${ }^{92}$ ".

88. "Le manuscrit Chevaleau. Seconde partie : sauver la Loire navigable ". Bulletin de l'Association des Amis du Musée de la Marine de Loire, (Châteauneuf-sur-Loire), n 75, septembre 1995, p. 36.

89. Arch. dép. de Maine-et-Loire, 118-S-1. Procès-verbal de la commission d'enquête sur le pont à construire entre Saumur et les Ponts-de-Cé, 1835. Document cité par DuBoIsRichIER, Amélie, op. cit., p. 148.

90. "Le manuscrit Chevaleau. 1839-1860. $1^{\text {re }}$ partie : l'homme, la rivière et les bateaux ", Bulletin de l'Association des Amis du Musée de la Marine de Loire (Châteauneuf-sur-Loire), $\mathrm{n}^{\circ} 74$, avril 1995 .

91. PoITRINEAU, Abel, « La Loire marchande, les trafics d'antan ", Une histoire de la Loire, 1986 , p. 98 et 105 .

92. "Le manuscrit Chevaleau. Seconde partie : sauver la Loire navigable ", Bulletin de l'Association des Amis du Musée de la Marine de Loire, (Châteauneuf-sur-Loire), n 75, septembre 1995, p. 36. 
Les voituriers tenaient toujours à préciser qu'ils procédaient " en la manière acoutumée sous corde ${ }^{93}$ ", d'autant que la déclaration du Roi d'avril 1703 prescrivait l'usage des ancres ainsi que le découplage des bateaux.

À la remontée il n'était pas nécessaire de détacher les bateaux d'un train pour franchir un pont. On faisait passer une ancre en amont, et on virait au guinda pour remonter le courant ${ }^{94}$. Un train de six ou sept bateaux pouvait alors franchir le pont d'un coup, sans être détachés. En d'autre lieux qu'aux Ponts-de-Cé, les premiers bateaux des trains pouvaient remonter leurs mâts une fois le pont passé, afin de bénéficier du vent. Mais selon Henri Chevaleau, il fallait attendre aux Ponts-de-Cé que tout le train soit passé : "Les élancements des bateaux pourraient faire chasser les ancres. " Souvent lors des crues les bateaux ne pouvaient passer les ponts faute d'espace suffisant sous les arches. Une manœuvre risquée, utilisée par un voiturier sous un pont d'Angers, consistait à faire entrer de l'eau dans le bateau pour abaisser la ligne de flottaison ${ }^{95}$.

Les ponts de Cé étaient le pont Saint-Aubin et le pont Saint-Maurille. Le pont Saint-Aubin reliait le bourg Saint-Aubin au nord de la Loire, vers Angers, à l'île forte située au milieu du fleuve. Il était coupé par la petite île de la Rabonnière. Le pont Saint-Maurille reliait l'île forte au bourg SaintMaurille au sud de la Loire. D'après Chevaleau, les voituriers préféraient passer en hiver sous le pont Saint-Aubin car il était jugé plus sûr. Mais l'été ce bras du fleuve était ensablé, alors que ses arches très basses devenaient vite infranchissables lors des crues. Un grande partie de l'année les voituriers étaient donc obligés de passer sous le pont Saint-Maurille. Chevaleau décrivit ce pont d'une manière effrayante. Les piles de pierre penchaient au-dessus des meilleures voies et les ruines des anciennes piles gênaient la navigation. Sur dix-neuf avaries aux Ponts-de-Cé dans les procès-verbaux, quatorze se sont produites au pont Saint-Maurille, trois au pont Saint-Aubin et deux ne sont pas localisées précisément.

D'après les procès-verbaux, les trois principales causes d'accident aux Ponts-de-Cé étaient le mauvais état du pont Saint-Maurille, les pieux et les fascines des mollets (pêcheries) débordant dans les voies et les tourbillons jetant les bateaux contre des piles. Là encore, les causes des avaries étaient

93. Un marinier parle de descendre en sonnette ou sonneil : " dessendant en sonnette avec ancre dont l'un des deux a bien passé et le second batteau neuf de bois de chesne decandant aussy en sonneil et ancre avec force monde cependant il auroit attrapé des pieux qui sont dans la voye des marchands " (5E 90/569, Fruslon, procès-verbal du $1^{\mathrm{er}}$ octobre 1749$)$.

94. La manœuvre est décrite plus en détail par Sébastien GODARD : " Le métier de marinier au XIX" siècle, apogée et déclin ", dans Archives d'Anjou. Un fleuve et des hommes : la Loire et ses affluents, une histoire tumultueuse, 2000.

95. "Ledit sieur Guery voyant que les eaux ne diminuoient point, voulant cependant obliger les marchands pour qui il etoit chargé à fait penetrer l'eau dans ses bateaux à la hauteur d'un pied pour avoir la facilité de passer par sous les ponts d'angers " (Arch. dép. de Maine-et-Loire, 5E 90/661, Foucher, procès-verbal du 23 novembre 1789. Passage d'un pont d'Angers le 20 novembre). 
multiples, et les tourbillons des flots comme les coups de vent jouaient souvent un rôle dans les accidents attribués aux pêcheries ${ }^{96}$. Au XIX ${ }^{\mathrm{e}}$ siècle, Henri Chevaleau insistait sur le courant qui arrivait de biais sur les arches du pont Saint-Maurille ${ }^{97}$ : à la descente comme à la remontée, les bateaux qui passaient sous ce pont étaient poussés vers une pile, puis vers la pile opposée quand ils avançaient plus loin dans la voie. Pour protéger son chaland lors du franchissement, un voiturier pouvait placer une toue du côté le plus dangereux ${ }^{98}$. Les voituriers rendaient souvent les tourbillons responsables de leurs accidents ${ }^{99}$, les attribuant aux grèves et anciens battis situés en amont. En effet, les ponts tombaient en ruine depuis longtemps, particulièrement celui de Saint-Maurille, et ils ne furent reconstruits qu'au milieu du XIX ${ }^{\mathrm{e}}$ siècle. L'aspect extérieur des ponts était catastrophique mais c'est sous l'eau que se cachaient les principaux dangers pour les voituriers. Les crèches des ponts, c'est-à-dire les rebords destinés à protéger les piles, reposaient sur des pieux. Ces pieux ou drajons étaient souvent abîmés - notamment à cause des glaces ${ }^{100}$ - et ils débordaient dans les voies. Quatre avaries sur dix-neuf étaient attribuées à des pieux de crèches, et deux autres à des crèches dans la voie, mais sans préciser si le problème venait des crèches en elles-mêmes ou des pieux qui les portaient.

Les pêcheries ou mollets étaient très nombreuses au XVIII ${ }^{\mathrm{e}}$ siècle. En 1770 , sur les vingt-deux piles du pont Saint-Maurille, quinze accueillaient des mollets à leur base ${ }^{101}$. Pour orienter le courant, les pêcheurs utilisaient des pieux qui faisaient l'objet de très nombreuses plaintes de la part des mari-

96. En 1779, les voituriers Legris et Delorme, d'Orléans, remontent de Nantes avec 6 ou 7 bateaux : " après avoir mis bas les mats et les voilles, ils ont virés au guindas pour remonter la rivière par la voye du moullin. Qu'ils avaient deja monté trois de leurs batteaux sans le plus leger accident, quand tout a coup un tourbillon de vant a soufflé avec tant de viollance quil a culbutté les derniers batteaux et poussé le quatrieme [...] sur un pieux cassé ». Pour sauver les autres bateaux, ils doivent couper les liens devant et derrière le bateau avarié (Arch. dép. de Maine-et-Loire, 5E 90/617, Fruslon, procès-verbal du 21 mars 1779).

97. À la remontée " le courant le dresse contre la pîle de bâbord et avec tant de force qu'il y est pour ainsi dire collé ". "Le manuscrit Chevaleau. Seconde partie : sauver la Loire navigable ". Bulletin de l'Association des Amis du Musée de la Marine de Loire (Châteauneuf-sur-Loire), $\mathrm{n}^{\circ} 75$, septembre 1995, page 38.

98. "Il aurait pris les précautions requises et necessaires pour se garantir des accidents, en mettant une toue au coté de son batteau pour l'empescher de heurter en dessendant soit contre les pilliers soit contre les pieux qui forment les voyes du pont " (Arch. dép. de Maine-et-Loire, 5E 90/607, Fruslon, procès-verbal du 18 avril 1768).

99. « La Loire dont l'impétuosité est extrème, roullant ses eaux fort inégallement auroit par un turbillon jeté le derrière vers plusieurs pilliers qui sont au dessous du pont qu'aussitost s'appercevant du dangers il auroit promptement pris un baston nommé arache car, à l'effet d'empescher son batteau de heurter : qu'ayant donc présenté son arache cor ou baston contre un pillier, ce même baston se serait cassé, qu'aussitost le basteau poussé par la rapidité de la rivière a donné contre quellesques fascinnes qui l'ont dechiré ". (Arch. dép. de Maine-et-Loire, 5E 90/605, Fruslon, procès-verbal du 18 mai 1765). 100. Les glaces étaient à l'origine de déplacements de pieux selon deux procès verbaux de 1729 et 1754 .

101. Arch. dép. de Maine-et-Loire, 5E 90/159, notaire Mabire de Juigné-sur-Loire, procèsverbal des pêcheries des ponts du 15 mai 1770 . 
niers. Ils les accusaient de déborder dans les voies, souvent de plusieurs pieds, ou de dévier le courant des autres voies ${ }^{102}$. Les deux professions s'opposaient pour l'utilisation des ponts. Ainsi, en 1737, le procureur de la compagnie des marchands fréquentant la rivière de Loire intervint pour demander la libération de la " voie de la ruine " où les fascines ne laissaient à l'eau qu'une ouverture de deux mètres, ce qui orientait le courant de biais sous l'arche voisine qui servait à la navigation. Il avait déjà fait déboucher cette voie à plusieurs occasions, et même l'année précédente, ce qui n'avait pas dissuadé les pêcheurs de boucher à nouveau la voie ${ }^{103}$... La litanie des plaintes des voituriers contre les pêcheurs se poursuivit jusqu'à la veille de la Révolution, mais au XIX ${ }^{\mathrm{e}}$ siècle Henri Chevaleau ne signalait plus les pêcheries qui avaient probablement été supprimées entre-temps.

\section{Les suites des avaries}

Le voiturier victime d'un accident était rapidement rejoint et assisté par les autres équipages naviguant sur la Loire. Les documents nous décrivent une activité intense sur le fleuve et rares étaient les voituriers qui avaient la malchance d'être entièrement seuls. Après une avarie, on déchargeait au plus vite la marchandise pour la mettre à l'abri, mais aussi pour accéder à la fracture, rendre le bateau plus manœuvrable en l'allégeant, et remonter la ligne de flottaison s'il n'avait pas encore coulé. Le blé mouillé était mis à sécher sur les rives, étalé sur des toiles ou dans des greniers, selon le temps et la saison ${ }^{104}$. On sortait les barriques d'une épave en les faisant rouler sur des poutres ou des bâtons de quartier ${ }^{105}$. Pour les trains de bois, qui risquaient de couler s'ils restaient trop longtemps dans l'eau, une méthode consistait à soutenir les radeaux avec des tonneaux ou des bateaux ${ }^{106}$.

\footnotetext{
102. «Etant sous l'arche dudit pont nommé le pont de saint maurille voulant eviter les ecueilles dangereux des fassines des motets qui debordent d'environ deux pied de chacun coste dans les voyes d'eau et empeschent la liberté de la navigation ce qui fait echoir journellement les bateaux en vin a til pû les eviter; ces miserables fassines par leurs debordements on dechiré et brisé fait faire naufrage au bateau " (Arch. dép. de Maine-etLoire, 5E 90/655, Foucher, procès-verbal du 11 juin 1774).

103. Arch. dép. de Maine-et-Loire, 5E 90/577, notaire Dubourg des Ponts-de-Cé, procèsverbal des voies du 12 octobre 1737 .

104. «Etoit un certain nombre de personnes dont nous a êtes impossible de compter qui avec leurs petites toues travailloient a transporter le dit blé qui etoit submergé d'un bord de la ditte rivière a l'autre, et le menoient sur le champtier de galairne pour le faire secher sur des bernes et voilles que nous y avons vu eparés " (Arch. dép. de Maine-etLoire, 5E19/7, notaire Dertron de Savennières, procès-verbal du 4 juin 1765).

105. « Le transport desd. Barriques s'est fait a la faveur de gros bois de quinze a dix huit pieds de long après que plusieurs se sont mis dans l'eau non obstant la rigueur du froid " (Arch. dép. de Maine-et-Loire, 5E 1/997, notaire Halnault d'Angers, procès-verbal du 26 novembre 1739 ).

106. En 1789 un sauvetage échoue, bien que les voituriers " ayent fait mettre le nombre de quinze tonneaux pour suporté les dits mairains [...] et ont pris deux bateaux un de
} 
Quand le bateau était endommagé mais n'avait pas coulé, le voiturier tentait de boucher la voie d'eau avec ce qu'il avait sous la main, souvent des vêtements, des draps ou de petites voiles appelées bernes ${ }^{107}$. L'équipage écopait, le temps de faire une réparation de fortune. On pouvait aussi utiliser d'autres bateaux pour maintenir le bateau avarié et l'empêcher de couler ${ }^{108}$. Quand la voie d'eau était trop importante, on tentait de placer le bateau près de la rive ou sur un haut-fond avant qu'il ne sombre, afin qu'il reste près de la surface ${ }^{109}$, car le plus difficile lors du sauvetage était de parvenir à remettre les bords hors d'eau de manière à pouvoir vider le bateau une fois la plaie réparée.

Pour remonter à la surface un bateau qui avait sombré, on utilisait plusieurs chalands, des cordages et des mâts ou parfois des bâtons de quartier. La manœuvre est décrite très succinctement dans la plupart des procès-verbaux mais certains extraits nous permettent malgré tout de la reconstituer ${ }^{110}$. On plaçait un ou deux bateaux de chaque côté de l'épave. Ensuite on passait en dessous et en travers des mâts ou des bâtons de quartiers que l'on reliait avec des cordages aux guindas des bateaux (les treuils situés à l'arrière qui servaient à dresser le mat). Puis on virait, c'est-à-dire que l'on faisait tourner les guindas, et l'épave était soulevée par les mâts. Cela n'était possible que si le bateau reposait à une faible profondeur. À plus de trois mètres de profondeur (10 pieds) l'épave paraissait irrécupérable. Le sauvetage nécessitait beaucoup de bras. La solidarité des voituriers et celle des riverains jouait pleinement, aiguisée par la promesse d'un bon

chaque bord pour soulager les dits mairains " (Arch. dép. de Maine-et-Loire, 5E 90/661, notaire Foucher des Ponts-de-Cé, procès-verbal du 8 mai 1789).

107. Ces bernes apparaissent fréquemment dans les inventaires et dans les procès-verbaux. Elles mesurent en général trois toiles, soit trois mètres soixante, et semblent servir aux petites toues de bord.

108. Un voiturier a "placé ses autres bateaux autour de lad. sapine pour empecher que tout le bled ne soit perdu " (Arch. dép. de Maine-et-Loire, 5E 42/58, notaire Deze de Saumur, procès-verbal du 31 octobre 1733).

109. En 1783, un voiturier " voyant la perte totale du sucre dont il etoit chargé, sil restoit dans l'endroit ou le bateau avoit rencontré le baton y ayant beaucoup d'eau, il derangea sa marche du ruault pour aller sur une greve ou il est maintenant un peu plus haut ou il a rencontré le baton sans quil soit passé une seule goutte d'eau par dessus les bords " (Arch. dép. de Maine-et-Loire, 5E16/273, notaire Rogeron de Saint-Mathurin, procès-verbal du 19 novembre 1783).

110. Arch. dép. de Maine-et-Loire, 5E 96/11, notaire Gaudin d'Ingrandes, procès-verbal du 16 janvier 1732 : « auroient monté deux bateaux a coste de celluy coulé avec force cordage et trois mats de bateaux aurois tire led. bateau de fond de leau ". Arch. dép. de Maine-et-Loire, 5E 90/575, notaire Dubourg des Ponts-de-Cé, procès-verbal du 13 décembre 1731 : « A emprunté quatre batteaux quatre mats pour virer et enlever la sapine du fond [...] il a esté obligé de prendre a louage des cordages pour virer dautant que les cordages servent aux liages des autres navires. " Arch. dép. de Maine-et-Loire, 5E 90/619, notaire Fruslon des Ponts-de-Cé, procès-verbal du 18 septembre 1781 : d'autres ont " approché deux de leurs batteaux pour lui aider à retirer du fond de l'eau celui qui malgré leur diligence venait d'y couller : qu'ayant effectivement viré avec le secours de plusieurs bastons qui portaient sur les deux batteaux qu'on avoit approché ils ont réussit à retirer de leau le bateau naufragé ". 
salaire. Les voituriers mobilisaient fréquemment 10 à 80 personnes, parfois sur plusieurs jours. À Juigné-sur-Loire en 1786 , un voiturier reçu l'aide de deux cents personnes grâce à l'appui du curé ${ }^{111}$.

Lorsque, suite à des collisions entre bateaux, d'autres voituriers étaient tenus pour responsables des avaries, les fautifs terminaient eux-mêmes la livraison ou, à défaut, remplaçaient les marchandises. Ils s'engageaient à faire réparer et livrer les bateaux avariés ou rachetaient les épaves. Quand seuls les voituriers et les marchands pour lesquels ils travaillaient étaient concernés, les marchands se retournaient rarement contre les voituriers et supportaient généralement seuls les pertes sur les marchandises. Dans les dépôts de bilan des marchands on trouve des pertes dues à des naufrages, mais rarement des dettes de voituriers. Le voiturier était tenu de payer des dommages et intérêts au marchand si le naufrage était arrivé par sa faute, mais encore fallait-il le prouver. Dans le cas contraire, après un naufrage arrivé par fortune de tems, les voituriers devaient, d'après la déclaration du roi de $1703^{112}$, abandonner leurs bateaux pour ne plus être considérés comme responsables des marchandises. Mais les marchands n'y avaient pas davantage intérêt que les voituriers, la marchandise étant alors "pêchée et tenue en justice à la conservation et aux frais de qui il appartiendra ", ce qui laissait augurer des dépenses importantes et des délais. Ils se montraient donc souvent relativement conciliants. Après les naufrages, les voituriers s'occupaient donc de sauver les marchandises et leurs bateaux, en mettant bien sûr l'accent sur les marchandises dans les procès-verbaux. Ils exigeaient généralement que les dépenses nécessaires pour le sauvetage des marchandises et la reprise de la livraison fussent remboursés par les marchands. Ces dépenses, avancées par les voituriers, comprenaient les salaires du personnel embauché sur place, mais aussi la location des greniers pour faire sécher le blé mouillé. Généralement on demandait aussi aux marchands de prendre en charge le renflouage du bateau et son rabillage, c'est-à-dire sa réparation. Les voituriers exigeaient

111. " Ils avoient requis le curé de publier dans l'eglise paroissialle de requerir tous ceux des paroissiens de juigné au nombre de cent cinquante ou deux cent pour autant qu'il sest de degager ledit batteau avarié moyennant une honnette retribution [le lendemain] avons trouvé lesd mariniers maitres et conducteurs dud train susd lesquels nous auraient dit que led batteau chargé et avarié ne pouvait etre tiré hors de l'eau et mis a sec sans une quantité de monde considerable, ayant sur son bord au vu de nous d. no.re environ quatre pieds d'eau au dessus des bords [...] lesd deux mariniers susd occuppés avec leur monde a preparer leur agrêts [...] sur les quatre heures environ est arrivé Mre Leau Archipretre de Juigné accompagné de son d. sr. vicaire, et d'environ deux cent paroissiens dud. Juigné sur Loire lesquels d'un commun accord s'etant mis a l'ouvrage, les srs. curé et vicaire aiant les premiers mis la main a l'oeuvre, chacun sest empressé de suivre leur exemple, et a force de travaux reiterés, lesd. mariniers sont parvenus a tirer sur le sable un bateau contenant suivant le rapport desd. mariniers et la lettre de voiture vingt six bariques de sucre brut; lequel d. batteau avec l'aide desd paroissiens aurait eté tiré a environ dix pieds de l'eau " (Arch. dép. de Maine-et-Loire, 5E 90/160, notaire Loizillon de Juigné-sur-Loire, procès-verbal du 30 septembre 1786).

112. Arch. dép. de Maine-et-Loire, C 22 navigation en Loire. Déclaration du Roi du 24 avril 1703. 
souvent d'être payés comme prévu pour leur voiture. Certains voulaient être dédommagés pour leur retard et même pour les objets personnels ou le matériel perdus lors du naufrage ${ }^{113}$. Reste à savoir s'ils obtenaient satisfaction pour ces demandes. Les voituriers avaient des atouts dans cette situation, car ils étaient les mieux à même de récupérer la marchandise et de lui faire reprendre sa route rapidement et à peu de frais. Les interlocuteurs des voituriers étaient, dans la majorité des cas, les marchands destinataires, déjà propriétaires des marchandises, mêmes si parfois on s'adressait à l'expéditeur quand il était proche. Seuls deux procès-verbaux signalent la venue sur place de marchands, mais il est vrai que les procèsverbaux étaient rédigés le jour même ou le lendemain des avaries ce qui laissait peu de temps pour les prévenir et leur permettre d'être présents. Dans les deux cas observés, les marchands acceptent de payer le sauvetage, paraissant surtout pressés de faire repartir au plus vite ce qui a pu être sauvé. Mais les marchands n'avaient intérêt à payer la réparation du bateau que si la livraison était encore possible. Les voituriers le savaient bien, et l'un d'eux déclarait : "Le monde qu'il mettera pour retirer les marchandises cy dessus avariées, touttes fois s'il est possible de les recouvrer seront aux frais de ceux à qui elles sont adressées. " Il acceptait donc l'idée de ne pas être remboursé en cas d'échec. Les voituriers restaient de toute façon perdants lors d'une avarie. Si la cargaison et le bateau étaient irrécupérables, le marchand perdait sa marchandise, et le voiturier son bateau, ce qui pouvait les ruiner tous deux.

La navigation en Loire, telle qu'elle apparaît à travers les procès-verbaux d'avaries, est délicate et irrégulière. Il est vrai qu'elle bénéficie d'atouts importants qui expliquent son extension et le rôle important qu'elle joue dans l'économie des provinces traversées : le fleuve est navigable loin en amont, les bateaux partis de Nantes croisent ceux de Nevers ou de Digoin et peuvent aller jusqu'à Paris par le Canal d'Orléans. De plus la navigation est facilitée entre Nantes et Orléans par les vents d'ouest dominants qui permettent de limiter le halage. Ainsi un bateau peut transporter jusqu'à trente tonnes de marchandises entre Nantes et Orléans avec un équipage limité à deux hommes. Mais la navigation sur la Loire est aussi dangereuse. Les bateaux sont instables. Leurs grandes voiles et l'absence de quilles les met à la merci d'un coup de vent de travers, c'est la rançon de leur légèreté et de leur faible tirant d'eau. Ils sont aussi d'une étonnante fragilité et coulent rapidement en cas de chocs avec les débris qui encombrent le chenal. D'autres dangers sont liés aux activités humaines : ponts vétustes aux arches étroites et basses, bâtons de quartiers laissés par des voituriers, pêcheries et moulins-bateaux avec lesquels les voituriers doivent bon gré mal gré partager le fleuve. Les voituriers disposent de moyens

113. " Proteste en outre ce pourvoir contre ainsy quil advisera bon estre afin de payement de ses hardes habits esquipages ustancilles de ses bateaux " (Arch. dép. de Maineet-Loire, 5E 90/433, notaire Berge des Ponts-de-Cé, procès-verbal du 24 janvier 1689). 
limités pour une navigation si compliquée. Il est difficile de louvoyer pour profiter au mieux du vent, le bateau ne s'y prête guère et le chenal est souvent trop étroit. La pautre ne sert qu'aux voyages vers l'amont, et les bâtons de quartier sont d'un maniement bien dangereux et délicat. Quant à l'usage des ancres, il paraît aléatoire et épisodique. En dehors des risques de naufrages, les voituriers sont à la merci des caprices du vent et du débit très irrégulier du fleuve qui peuvent provoquer des retards très importants. Ces retards engendrent une incertitude pour le commerce. Les contraintes de la navigation en Loire, son irrégularité surtout, contribuèrent à son déclin quand au milieu du XIX ${ }^{\mathrm{e}}$ siècle le transport fluvial se retrouva en concurrence avec le transport ferroviaire, régulier, rapide et qui ne dépendait pas d'un compromis toujours précaire avec les éléments naturel.

\section{Annexe - Localisation des avaries et des retards}

Nous avons surtout consulté les archives de notaires résidant entre Angers et Saumur, et en particulier aux Ponts-de-Cé. Le premier nombre correspond au total des procès-verbaux, le second entre parenthèses aux seuls procès-verbaux de retards

$\begin{array}{lrlr}\text { Angers } & 3(1) & \text { Saint-Lambert des Levées } & 2 \\ \text { Bouchemaine } & 4 & \text { Montsoreau } & 3 \\ \text { La Pointe } & 1(1) & \text { Varennes/Loire } & 1 \\ & & \text { Parnay } & 1 \\ \text { D'Angers à Candes } & \mathbf{1 6 6} & \text { Villebernier } & 9 \\ \text { Saint-Gemmes } & 7 & \text { Savigny } & 1 \\ \text { Ponts-de-Cé } & 49(10) & \text { (en amont de Candes, sur la Vienne) } \\ \text { Sorges } & 2 & & \\ \text { Juigné } & 6 & \text { De Nantes à Angers } & \mathbf{5 8} \\ \text { Daguenière } & 4 & \text { Anetz } & 1 \\ \text { Blaison } & 1 & \text { Behuard } & 3 \\ \text { La Bohalle } & 14 & \text { Chalonnes } & 9 \\ \text { Saint-Mathurin } & 21(2) & \text { Ingrandes } & 17(6) \\ \text { Saint-Remy } & 2 & \text { Montrelais } & 3 \\ \text { Saint-Maur } & 1 & \text { Possonnière } & 1 \\ \text { Le Thoureil } & 1 & \text { Rochefort } & 2 \\ \text { Les Rosiers } & 14(1) & \text { Savennières } & 13 \\ \text { Bessé } & 4 & \text { Saint-Georges-sur-Loire } & 1 \\ \text { Gennes } & 3 & \text { Champtocé } & 1 \\ \text { Saint-Clément-des-Levées } & 5(1) & \text { La Meilleraie } & 1 \\ \text { Cunault } & 2 & \text { Montjean } & 2 \\ \text { Chenehutte } & 1 & \text { Saint-Florent-le-Vieil } & 1 \\ \text { Saint-Martin-de-la-Place } & 5(1) & \text { Varades } & 1 \\ \text { Saumur } & 6(2) & \text { Drain } & 1 \\ \text { Saint-Hilaire } & 1 & \text { Ancenis } & 1 \\ & & & \end{array}$




\section{Bibliographie}

BEAUDOUIN, François, "La marine de Loire et son chaland ", Bulletin de l'Association des Amis du Musée de la Batellerie, $\mathrm{n}^{\circ}$ 12, juin 1984.

BILLACoIS, François, "La batellerie de la Loire au XVII ${ }^{\mathrm{e}}$ siècle ", Revue d'Histoire Moderne et Contemporaine, 1964, p. 163-190.

Chaussard, Paul, La Marine de Loire, Cahiers du Bourbonnais, Éd. Charroux, 1998, $176 \mathrm{p}$.

Chevaleau Henri, "Le manuscrit Chevaleau. 1839-1860. $1^{\text {re }}$ partie : "L'homme, la rivière et les bateaux ", Bulletin de l'Association des Amis du Musée de la Marine de Loire, $\mathrm{n}^{\circ}$ 74, avril 1995. $2^{\mathrm{e}}$ partie, "Sauver la Loire navigable. 1807-1845 ", $\mathrm{n}^{\circ} 75$, septembre 1995.

—, "Les transports de 1610 à nos jours ", 104e congrès national des Sociétés savantes, Bordeaux, 1979, Paris, Bibliothèque Nationale, 1980, 452 p.

Creuze-Latouche, Jacques-Antoine, Description topographique du district de Chatelleraud, département de la Vienne, avec l'exposition de la nature de son sol, de ses diverses productions, de l'état actuel de son commerce \& de son agriculture, des observations sur le caractère \& les moeurs de ses habitans, \& une carte du pays/par M. Creuzé-Latouche, P. J. B. Guimbert éd., Châtellerault 1790, réimprimé Maxwell, 1992, x-119 p.

DeZAnNEau, Bénédicte, Les hommes et la Loire à Saint-Clément-des-Levées. 1750-1789, Angers, Maulévrier, 1990, 206 p.

Dıon, Roger, "Orléans et l'ancienne navigation de la Loire ", Annales de géographie, 1938, p. 128-154.

DuBoIs-RICHIR, Amélie, La Loire au XIX siècle : le fleuve et ses riverains de Saumur à Bouchemaine, Thèse d'histoire, 4 vol., université d'Angers, 2001.

Dumas, La Généralité de Tours au XVIII siècle (1766-1783), Paris, 1894, 437 p. Extrait des Mémoires de la société archéologique de Touraine, t. 39.

FraYsSE, Jeanne, Camille, Loire angevine et Maine, Cholet, 1974, 175 p.

MARCILHACY, Christiane, "Les mariniers et la marine de la Loire vus du port de Châteauneuf-sur-Loire ", Arts et Traditions populaires, 1960.

"Mémoire concernant la navigation des Rivières sur lesquelles les Entrepreneurs de la voiture des sels font le fournissement des Greniers de Grandes Gabelles " (Archives Nationales : G1/97). Cité dans " La navigation sur la Loire et ses affluents vers 1785 », publié dans les Annales de Bretagne (36, 1, 1924-1925) et dans L'Anjou historique (janvier 1927).

MERLET, Monique, Le péage de la cloison d'Angers (XVe-XVIII siècle), contribution à l'étude du trafic de la Loire à la fin de l'Ancien Régime, thèse de $3^{\mathrm{e}}$ cycle, École nationale des chartes, 1967.

POITRINEAU, Abel, " La Loire marchande, les trafics d'antan ", dans Une histoire de la Loire, Paris, Ramsay, 1986, 413 p.

—, "L'économie du transport fluvial. Une esquisse ", Revue historique, CCLXXXV/1, 1991.

PORT, Célestin, "Les inondations dans le département de Maine-et-Loire ", Revue de l'Anjou, 1856. Repris dans Questions Angevines, 1884.

REMOND, André, Les prix des transports marchands de la révolution au Premier Empire, série "Étude sur la circulation marchande en France aux XVIII et XIX ${ }^{\mathrm{e}}$ siècles ", M. Rivière, coll. « Bibliothèque d'histoire économique et sociale ", 1956, 112 p. 


\section{RÉSUMÉ}

Au XVIII ${ }^{\mathrm{e}}$ siècle la navigation sur la Loire était dangereuse. Les voituriers par eau étaient souvent victimes d'avaries ou de naufrages qui entraînaient la rédaction par des notaires de procès-verbaux destinés à se couvrir auprès des marchands. Nous en avons étudié plus de 200 rédigés en Anjou entre 1689 à 1791. Ils sont une source abondante d'informations sur la navigation en Loire. Recoupés avec d'autres documents, ils permettent de mieux connaître les conditions de navigation, les dangers encourus et les conséquences des accidents, y compris les méthodes utilisées pour remettre à flot les bateaux naufragés.

Bien que rédigés par des notaires, ils présentent l'avantage de laisser transparaître le point de vue des navigateurs. Les voituriers dressent à travers ces témoignages un tableau vivant de la navigation en Loire, riche de détails vécus qui échapperaient à des témoins extérieurs. Ces documents sont d'autant plus précieux que les voituriers de la Loire ont laissé bien peu de témoignages écrits sur leur métier.

\section{ABSTRACT}

During the $18^{\text {th }}$ Century the navigation on the Loire was pretty dangerous. The bargemen were often victims of damage or shipwrecks that induced the compilation by notaries of verbal processes destined to cover themselves against the merchants. We have since studied more than 200 testimonies written in Anjou between 1689 and 1791. These are an abundant source of information concerning the navigation on the Loire. Gathered with other documents, they allow a better knowledge of the navigation conditions, the incurred dangers and the consequences of the accidents, including the methods used to put back afloat the shipwrecked boats. Although written down by notaries, they present the advantage of showing the navigators' point of view. The bargmen erect through these testimonies a living picture of the navigation in Loire, rich of details that would not understand exterior witnesses. These documents are even more precious than the bargemen of the Loire left a few written testimonies about their craftship. 